\title{
Scattering of electromagnetic waves by rough interfaces and inhomogeneous layers
}

Article

Published Version

Chandler-Wilde, S. N. and Zhang, B. (1999) Scattering of electromagnetic waves by rough interfaces and inhomogeneous layers. SIAM Journal on Mathematical Analysis, 30 (3). pp. 559-583. ISSN 0036-1410 doi: https://doi.org/10.1137/S0036141097328932 Available at https://centaur.reading.ac.uk/32652/

It is advisable to refer to the publisher's version if you intend to cite from the work. See Guidance on citing.

Published version at: http://dx.doi.org/10.1137/S0036141097328932

To link to this article DOI: http://dx.doi.org/10.1137/S0036141097328932

Publisher: Society for Industrial and Applied Mathematics

All outputs in CentAUR are protected by Intellectual Property Rights law, including copyright law. Copyright and IPR is retained by the creators or other copyright holders. Terms and conditions for use of this material are defined in the End User Agreement.

www.reading.ac.uk/centaur 
Central Archive at the University of Reading

Reading's research outputs online 


\title{
SCATTERING OF ELECTROMAGNETIC WAVES BY ROUGH INTERFACES AND INHOMOGENEOUS LAYERS*
}

\author{
SIMON N. CHANDLER-WILDE ${ }^{\dagger}$ AND BO ZHANG ${ }^{\ddagger}$
}

\begin{abstract}
We consider a two-dimensional problem of scattering of a time-harmonic electromagnetic plane wave by an infinite inhomogeneous conducting or dielectric layer at the interface between semi-infinite homogeneous dielectric half-spaces. The magnetic permeability is assumed to be a fixed positive constant. The material properties of the media are characterized completely by an index of refraction, which is a bounded measurable function in the layer and takes positive constant values above and below the layer, corresponding to the homogeneous dielectric media. In this paper, we examine only the transverse magnetic (TM) polarization case. A radiation condition appropriate for scattering by infinite rough surfaces is introduced, a generalization of the Rayleigh expansion condition for diffraction gratings. With the help of the radiation condition the problem is reformulated as an equivalent mixed system of boundary and domain integral equations, consisting of second-kind integral equations over the layer and interfaces within the layer. Assumptions on the variation of the index of refraction in the layer are then imposed which prove to be sufficient, together with the radiation condition, to prove uniqueness of solution and nonexistence of guided wave modes. Recent, general results on the solvability of systems of second kind integral equations on unbounded domains establish existence of solution and continuous dependence in a weighted norm of the solution on the given data. The results obtained apply to the case of scattering by a rough interface between two dielectric media and to many other practical configurations.
\end{abstract}

Key words. scattering, integral equation, inhomogeneous medium, Helmholtz equation

AMS subject classifications. 35J05, 35L05, 45E10, 78A45

PII. S0036141097328932

1. Introduction. Consider a time harmonic electromagnetic plane wave incident on a layer of some inhomogeneous, isotropic, conducting, or dielectric material in $\mathbf{R}^{3}$. The media, above and below the layer, consist of some homogeneous dielectric materials. Adopting Cartesian axes $0 x_{1} x_{2} x_{3}$, we assume throughout that the material is invariant in the $x_{3}$ direction. Thus, in effect, the problem geometry is twodimensional. Further, we assume that the magnetic permeability is a fixed positive constant. The material properties of the media are then characterized completely by an index of refraction, dependent on the permittivity and conductivity, which is assumed to be a bounded measurable function in the layer and takes positive constant values above and below the layer. The scattering problem is to study the electromagnetic field distributions.

In this paper we formulate first the scattering problem as a boundary value problem for the reduced wave equation (Helmholtz equation), using a radiation condition recently introduced for problems of scattering by infinite one-dimensional rough surfaces and interfaces $[4,5,7,8,9]$, which is a generalization of the usual radiation condition used in the study of plane wave diffraction by one-dimensional periodic gratings (see, e.g., $[23,1,2,4,19,20,25]$ ). Next, in section 3 , we derive a novel

${ }^{*}$ Received by the editors October 20, 1997; accepted for publication (in revised form) July 27, 1998; published electronically March 29, 1999. This work was supported by the UK Engineering and Physical Sciences Research Council under grant GR/K24406.

http://www.siam.org/journals/sima/30-3/32893.html

$\dagger$ Department of Mathematics and Statistics, Brunel University, Uxbridge UB8 3PH, UK (Simon. Chandler-Wilde@ brunel.ac.uk).

${ }^{\ddagger}$ School of Mathematical and Information Sciences, Coventry University, Coventry CV1 5FB UK (b.zhang@coventry.ac.uk). 
integral equation formulation of the problem, as a system of coupled second-kind domain and boundary integral equations, over the layer and over interfaces within the layer, and establish that this formulation is equivalent to the formulation as a boundary value problem. The radiation condition imposed does not rule out guided waves, localized in the inhomogeneous layer, which are thus solutions of the homogeneous boundary value problem and the homogeneous integral equation formulation. From section 4 onward we make restrictions on the variation of the index of refraction in the layer. Under these restrictions we establish, in section 4, an a priori inequality satisfied by any solution. Using this inequality, a key lemma from [8], and extensions of arguments in $[7,31]$, uniqueness results and hence conditions for the nonexistence of guided wave modes are established in section 5 . In section 6 , existence of solution is established by employing a novel form of Fredholm alternative based on general results on the solvability of systems of integral equations on unbounded domains in [10].

The assumptions we impose on the index of refraction from section 4 onward are satisfied in many practical cases. In particular, the results obtained apply to the case of scattering by a rough interface between two dielectric media and apply to scattering by a homogeneous layer having rough interfaces, with the media above and below, provided that the wavenumbers in the layer $\left(k^{*}\right)$, and in the media above $\left(k_{+}\right)$and below $\left(k_{-}\right)$, satisfy either that $\Im k^{*}>0$ or that $\max \left(k_{-}, k_{+}\right)>k^{*}$. For a precise statement of the cases covered see section 2. Our conclusion that no guided waves exist if $\max \left(k_{-}, k_{+}\right)>k^{*}$ or $\Im k^{*}>0$ is in agreement with explicit analytical calculations of guided wave modes for the case of plane interfaces between the layer and the media [24].

Integral equation methods have been used widely in the theoretical and numerical study of wave scattering by finite obstacles or local inhomogeneities (see, e.g., [12, 13] and the references quoted there). More recently they have been employed to study scattering by periodic structures $[11,17,19,20,22]$ and by a nonstratified local inhomogeneity in a stratified medium [30]. Integral equation formulations have also been used extensively in computations of wave scattering by infinite one- and twodimensional rough surfaces and interfaces (see, e.g., [26, 14, 21, 29] and the references quoted there), but little attention appears to have been paid in the literature to their mathematical justification (a recent exception is [15]).

This present paper is intended, in part, as a contribution to the mathematical analysis of rough surface scattering problems and of the well-posedness of their formulation as integral equations. It is related, in terms of results and methods of argument, to recent studies of scattering of a wave incident from a homogeneous half-space onto an inhomogeneous impedance plane [5]; of electromagnetic waves by a one-dimensional perfectly conducting rough surface $[6,8]$; of electromagnetic waves by an inhomogeneous conducting or dielectric layer on a perfectly conducting plate [7]; and of acoustic waves by an inhomogeneous layer on a rigid plate [31]. In particular, the present study is closest to this last paper [31] in that the existence proof depends on the same novel results on the solvability of systems of weakly singular second-kind integral equations on unbounded domains. However, the whole space problem considered here requires a substantially more elaborate uniqueness proof and integral equation formulation, related to the presence of transmitted as well as reflected waves, in contrast to the half-plane problems considered in $[5,6,8,7,31]$. Moreover, in these latter papers integral equation formulations using half-plane Green's functions appear natural: as proposed here, the formulation of the whole-space problem as a system of integral 
equations in overlapping half-planes, using half-plane Green's functions, is surprising but proves powerful in establishing uniqueness and existence results.

This paper can also be viewed as a generalization of the results of Bonnet-Bendhia and Starling [3] and Strycharz [25], who study plane wave scattering by an inhomogeneous periodic layer. In fact, our uniqueness results derive in part from those of Bonnet-Bendhia and Starling [3] and Strycharz [25], and include some of their results for a periodic layer as special cases; however, note that our results are obtained without an a priori assumption of quasi periodicity of the scattered field. We note also that our existence arguments, based on integral equation methods, differ from the variational methods used in [3] and [25] which appear restricted to the periodic case. We further point out that while integral equation-based existence proofs are common (and more straightforward) in the periodic, diffraction grating case (e.g., [19, 11]), they usually fail for a discrete set of combinations of grating period and angle of incidence at which the integral equation formulation is undefined. Our results show that, at least in the two-dimensional case, this problem can be avoided by use of a halfplane rather than a whole-space Green's function in the integral equation formulation. A finite element method for the case of plane wave scattering by an inhomogeneous periodic layer is analyzed in [1].

We remark that aside from the theoretical use to which the formulation is put in this paper, we anticipate that the novel integral equation formulation we derive in section 3 may also be of value for numerical computation. We point out that the integral operators in our formulation are exclusively of convolution type or are products of convolution and multiplication operators so that, after discretization, the matrix vector multiplications required in an iterative solution scheme can be performed efficiently using the FFT (see [27, pp. 109-111] and [28]). We further point out that, even for the simple case of a single interface between two dielectric media, for which a boundary integral equation formulation on the interface is usual (avoiding domain integrals), a recently successful numerical algorithm involves imbedding the one-dimensional boundary curve in a two-dimensional grid so that FFT techniques can be applied [29].

We conclude this section by introducing some notations used throughout. For $h \in \mathbf{R}$, define $\Gamma_{h}=\left\{x=\left(x_{1}, x_{2}\right) \in \mathbf{R}^{2} \mid x_{2}=h\right\}$ and $U_{h}=\left\{x \in \mathbf{R}^{2} \mid x_{2}>h\right\}$. Set $E_{h}^{H}=U_{h} \backslash \bar{U}_{H}$ for $H>h$, and write $U, \Gamma$, and $E_{H}$ for $U_{0}, \Gamma_{0}$, and $E_{0}^{H}$, respectively. Define $D_{A}=\left\{x \in \mathbf{R}^{2}|| x_{1} \mid<A\right\}, A>0$, and $\Gamma_{h}(A)=\Gamma_{h} \cap D_{A}, E_{h}^{H}(A)=E_{h}^{H} \cap D_{A}$. For $G \subset \mathbf{R}^{2}$ let $B C(G)$ denote the space of bounded continuous functions defined on $G$. For $v \in C^{1}\left(\mathbf{R}^{2}\right)$ denote by $\partial_{j} v, j=1,2$, the derivative $\partial v(x) / \partial x_{j}$. Finally, for $A>0, x \in \mathbf{R}^{2}$, let $B_{A}(x)=\left\{y \in \mathbf{R}^{2}|| y-x \mid<A\right\}$.

2. The scattering problem and radiation conditions. Let us assume that $\mathbf{R}^{3}$ is filled with an inhomogeneous, isotropic, conducting, or dielectric medium of electric permittivity $\epsilon>0$, magnetic permeability $\mu>0$, and electric conductivity $\sigma \geq 0$. Suppose that the medium is nonmagnetic, i.e., the magnetic permeability $\mu$ is a fixed constant in $\mathbf{R}^{3}$, and suppose that the fields are source free. Then the electromagnetic wave propagation is governed by the time-harmonic Maxwell equations (time dependence $\exp (-i \omega t)$ with frequency $\omega>0$ )

$$
\begin{aligned}
\nabla \times E-i \omega \mu H & =0, \\
\nabla \times H+(i \omega \epsilon-\sigma) E & =0,
\end{aligned}
$$

where $E$ and $H$ are the electric field and magnetic field, respectively. In this paper, it is assumed that the medium is invariant in the $x_{3}$ direction, i.e., $\epsilon=\epsilon(x)$ and $\sigma=\sigma(x)$ 
with $x=\left(x_{1}, x_{2}\right) \in \mathbf{R}^{2}$. Also, we restrict ourselves to the transverse magnetic (TM) polarization case; that is, the electric field $E$ is assumed to point along the $x_{3}$ axis. Let $E=(0,0, u)$, where $u=u(x)$ is a scalar function. Then it follows from the Maxwell equations (2.1)-(2.2) that $u$ satisfies the reduced wave equation

$$
\Delta u+k^{2} u=0 \text { in } \mathbf{R}^{2},
$$

where $\Delta$ is the Laplacian in $\mathbf{R}^{2}$ and $k^{2}=\omega^{2} \mu \epsilon[1+i \sigma /(\omega \epsilon)]$ so that $\Im\left(k^{2}\right) \geq 0$.

Additionally we make the following assumptions on $k$ throughout:

(A1) $k \in L_{\infty}\left(\mathbf{R}^{2}\right)$.

(A2) There are positive constants $B, k_{+}$, and $k_{-}$such that $k(x)=k_{+}$for $x \in U_{B}$, $=k_{-}$for $x \in \mathbf{R}^{2} \backslash \bar{U}$.

These two assumptions are sufficient (together with the radiation conditions we introduce below) to derive, in section 3 , an equivalent integral equation formulation of the problem. In sections 4 and 5 we address the question of uniqueness of solution which is related to the question of existence or otherwise of guided wave solutions of the homogeneous problem.

We remark that the radiation conditions we will impose will ensure that the scattered field does not contain a downward propagating component and that the transmitted wave does not contain an upward propagating component but (in common with the usual radiation condition for plane wave incidence on periodic gratings) will not rule out solutions of the homogeneous problem which are guided waves localized in the inhomogeneous layer. (See Theorem A.1 in the appendix, where a precise definition of a guided wave in this context is given.) Thus, to prove any uniqueness result, we will have to impose additional conditions (on $k$ ) which rule out guided waves. In other words (and more positively), any uniqueness proof will simultaneously establish conditions for the nonexistence of guided waves.

The additional requirements for our uniqueness proof (sections 4 and 5) and for proving the existence of solution (section 6) are that assumption (A3) is satisfied or that both assumptions (A4) and (A5) below are satisfied.

(A3) There exist constants $\lambda_{1}, \lambda_{2}, \eta$, and $\rho$, with $\lambda_{1}>0,1>\lambda_{2}>0$ and $0 \leq$ $\eta<\rho \leq B$ such that $\Im\left(k^{2}(x)\right) \geq \lambda_{1}$ for almost all $x \in E_{\eta}^{\rho}, \Im\left(k^{2}(x)\right) \geq$ $\lambda_{2}\left|k^{2}(x)-k_{+}^{2}\right|$ for almost all $x \in E_{\eta}^{B}$, and $\Im\left(k^{2}(x)\right) \geq \lambda_{2}\left|k^{2}(x)-k_{-}^{2}\right|$ for almost all $x \in E_{0}^{\rho}$.

(A4) There exists $\beta \in \mathbf{R}$ such that $\Re\left(k^{2}(x)\right)$ is monotonic nondecreasing in $U_{\beta}$ and monotonic nonincreasing in $\mathbf{R}^{2} \backslash U_{\beta}$ as $x_{2}$ increases: precisely, for all $h>0$, where $e_{2}=(0,1), \Re\left[k^{2}\left(x+e_{2} h\right)\right] \geq \Re\left[k^{2}(x)\right]$ for almost all $x \in U_{\beta}$ and $\Re\left[k^{2}\left(x-e_{2} h\right)\right] \geq \Re\left[k^{2}(x)\right]$ for almost all $x \in \mathbf{R}^{2} \backslash \bar{U}_{\beta}$.

Let $\tilde{k}(x)=k_{+}$for $x_{2} \geq \beta,=k_{-}$for $x_{2}<\beta$. Then Assumption (A4) implies that $\Re\left[k^{2}(x)\right] \leq \tilde{k}^{2}(x)$ for almost all $x \in \mathbf{R}^{2}$.

(A5) There are constants $\lambda_{3}, \eta$, and $\rho$, with $\lambda_{3}>0$ and $0 \leq \eta<\rho \leq B$, such that $\Re\left[k^{2}(x)\right] \leq \tilde{k}^{2}(x)-\lambda_{3}$ for almost all $x \in E_{\eta}^{\rho}$.

We can write (A4) succinctly as

$$
\left(x_{2}-\beta\right) \partial_{2}\left(\Re\left(k^{2}\right)\right) \geq 0
$$

in a distributional sense in $\mathbf{R}^{2}$ (cf. Bonnet-Bendhia and Starling [3, equation (3.34)] and [7]). If (A4) and (A5) hold with $\beta<\rho$, then it must be the case that $\Re\left[k^{2}(x)\right] \leq$ $k_{+}^{2}-\lambda_{3}$ for almost all $x \in E_{\beta}^{\rho}$, while if $\beta>\eta$, then $\Re\left[k^{2}(x)\right] \leq k_{-}^{2}-\lambda_{3}$ for almost all $x \in E_{\eta}^{\beta}$. 
To clarify the above assumptions we list some important practical cases in which they are satisfied:

(i) Suppose that $k_{+} \neq k_{-}$and, for some $f \in L_{\infty}(\mathbf{R})$, that $k(x)=k_{+}, x_{2}>f\left(x_{1}\right)$, $=k_{-}, x_{2}<f\left(x_{1}\right)$, and assume without loss of generality that $\epsilon \leq f\left(x_{1}\right) \leq B-\epsilon$ for some $\epsilon>0, B>\epsilon$. Then (A1), (A2), (A4), (A5) are satisfied, with $0=\beta=\eta, \rho=\epsilon$, if $k_{-}<k_{+}, B-\epsilon=\eta, B=\beta=\rho$, if $k_{-}>k_{+}$.

(ii) Suppose that $k^{*}>0$ and, for some $f_{+}, f_{-} \in L_{\infty}(\mathbf{R})$, with $f_{-} \leq f_{+}$, that $k(x)=k_{+}, x_{2}>f_{+}\left(x_{1}\right),=k_{-}, x_{2}<f_{-}\left(x_{1}\right),=k^{*}, f_{-}\left(x_{1}\right)<x_{2}<f_{+}\left(x_{1}\right)$. Suppose further without loss of generality that, for some $B>\epsilon>0, \epsilon \leq f_{-}\left(x_{1}\right) \leq f_{+}\left(x_{1}\right) \leq$ $B-\epsilon, x_{1} \in \mathbf{R}$. Then assumptions (A1) and (A2) are satisfied and so are assumptions (A4) and (A5) in the following cases: (a) $k_{-}<k^{*}<k_{+}$(set $\left.\beta=\eta=0, \rho=\epsilon\right)$; (b) $k_{-}>k^{*}>k_{+}($set $\eta=B-\epsilon, \beta=\rho=B)$; (c) $k^{*}<k_{+}, k^{*}<k_{-}$provided, for some $0<\eta<\rho<B, k(x)=k^{*}, x \in E_{\eta}^{\rho}$, and $\eta \leq \beta \leq \rho$.

(iii) Suppose that $\Im k^{*}>0$ and that, for some $0<\eta<\rho<B$, and disjoint open sets $S_{+}, S$, and $S_{-}$, with $\overline{S_{+}} \cup \bar{S} \cup \overline{S_{+}}=\mathbf{R}^{2}$ and $E_{\eta}^{\rho} \subset S, U_{B} \subset S_{+}$, and $\mathbf{R}^{2} \backslash \bar{U} \subset S_{-}$,

$$
k(x)= \begin{cases}k_{+}, & x \in S_{+}, \\ k^{*}, & x \in S_{-}, \\ k_{-}, & x \in S_{-} .\end{cases}
$$

Then (A1)-(A3) are satisfied.

We mention one simple example not covered by the above assumptions. In the case $k \equiv k_{+}$assumptions (A1), (A2), and (A4) are satisfied (with $k_{-}=k_{+}$), but assumption (A5) is not satisfied. Thus, the uniqueness results established in section 4 do not apply, and indeed, our problem as formulated will not have a unique solution in this case as is shown by the simple example $u(x)=\exp \left( \pm i k_{+} x_{1}\right)$, which satisfies (2.3) with $k \equiv k_{+}$and, by Remark 2.3 below, the radiation conditions (2.4) and (2.5).

Let $u^{\mathrm{i}}(x)=\exp \left(i k_{+} x \cdot \alpha\right)$ be the time-harmonic incoming plane wave incident from $U_{B}$ on the finite inhomogeneous layer $E_{B}$, where $x \in \mathbf{R}^{2}, \alpha=(\cos \theta,-\sin \theta) \in \mathbf{R}^{2}$, and $\theta \in(0, \pi)$ is the incident angle. We are interested in finding the total field $u$ satisfying the reduced wave equation (2.3).

In order to determine the physical solution $u$, a radiation condition as $x_{2}$ tends to infinity has to be imposed on the scattered field $u^{\mathrm{s}}=u-u^{\mathrm{i}}$ in $U_{B}$; that is, the scattered field $u^{\mathrm{s}}$ should behave as an outgoing wave as $x_{2} \rightarrow+\infty$. Similarly, the transmitted field $u$ in $\mathbf{R}^{2} \backslash U$ should behave as an outgoing wave as $x_{2} \rightarrow-\infty$. The standard Sommerfeld radiation condition is not appropriate in this context as we cannot expect that the scattered and the transmitted fields will decay at infinity. We will use a radiation condition proposed in [5] and utilized recently in $[7,8]$ and [31], which we will usefully relate to the Sommerfeld radiation condition. To this end we introduce the following definitions.

Definition 2.1. Given a domain $G \subset \mathbf{R}^{2}$ and $k_{*}>0$, call $v \in C^{2}(G) \cap L_{\infty}(G)$ a radiating solution of the Helmholtz equation for wavenumber $k_{*}$ in $G$ if $\Delta v+k_{*}^{2} v=0$ in $G$ and

$$
\begin{aligned}
v(x) & =O\left(r^{-1 / 2}\right), \\
\frac{\partial v(x)}{\partial r}-i k_{*} v(x) & =o\left(r^{-1 / 2}\right),
\end{aligned}
$$

as $r=|x| \rightarrow \infty$, uniformly in $x /|x|$.

Let $\Phi\left(x, y ; k_{ \pm}\right)$denote the free-space Green's function for $\Delta+k_{ \pm}^{2}$; that is,

$$
\Phi\left(x, y ; k_{ \pm}\right)=\frac{i}{4} H_{0}^{(1)}\left(k_{ \pm}|x-y|\right), \quad x, y \in \mathbf{R}^{2}, \quad x \neq y,
$$


with $H_{0}^{(1)}$ being the Hankel function of the first kind of order zero.

Definition 2.2. Given a domain $G \subset \mathbf{R}^{2}$, say that $v_{+}: G \rightarrow \mathbf{C}$ satisfies the upward propagating radiation condition (UPRC) for wavenumber $k_{+}$in $G$ if, for some $H \in \mathbf{R}$ and $\phi_{+} \in L_{\infty}\left(\Gamma_{H}\right)$, it holds that $U_{H} \subset G$ and

$$
v_{+}(x)=2 \int_{\Gamma_{H}} \frac{\partial \Phi\left(x, y ; k_{+}\right)}{\partial y_{2}} \phi_{+}(y) d s(y), \quad x \in U_{H} ;
$$

and say that $v_{-}: G \rightarrow \mathbf{C}$ satisfies the downward propagating radiation condition (DPRC) for wavenumber $k_{-}$in $G$ if, for some $h \in \mathbf{R}$ and $\phi_{-} \in L_{\infty}\left(\Gamma_{h}\right)$, it holds that $\mathbf{R}^{2} \backslash \bar{U}_{h} \subset G$ and

$$
v_{-}(x)=-2 \int_{\Gamma_{h}} \frac{\partial \Phi\left(x, y ; k_{-}\right)}{\partial y_{2}} \phi_{-}(y) d s(y), \quad x \in \mathbf{R}^{2} \backslash \bar{U}_{h} .
$$

Note that the existence of the integrals in (2.4) and (2.5) for arbitrary $\phi_{+} \in L_{\infty}\left(\Gamma_{H}\right)$ and $\phi_{-} \in L_{\infty}\left(\Gamma_{h}\right)$ is ensured by the bound which follows from the asymptotic behavior of the Hankel function for small and large argument,

$$
\left|\frac{\partial \Phi\left(x, y ; k_{ \pm}\right)}{\partial y_{2}}\right| \leq C\left|x_{2}-y_{2}\right|\left(|x-y|^{-2}+|x-y|^{-3 / 2}\right), \quad x, y \in \mathbf{R}^{2}, \quad x \neq y
$$

which holds for some constant $C>0$ dependent only on $k_{ \pm}$.

The next lemma states properties of the upward propagating radiation condition needed later and, in particular, shows that, for $h \in \mathbf{R}$, a radiating solution for wavenumber $k_{*}$ in $U_{h}\left(\mathbf{R}^{2} \backslash \bar{U}_{h}\right)$ satisfies the UPRC (DPRC) for wavenumber $k_{*}$. We first remark that the DPRC can be expressed, through reflection, in terms of the UPRC.

Remark 2.1. For $x=\left(x_{1}, x_{2}\right) \in \mathbf{R}^{2}$ let $x^{\prime}=\left(x_{1},-x_{2}\right)$, and for $G \subset \mathbf{R}^{2}$ let $G^{\prime}=\left\{x^{\prime} \mid x \in G\right\}$. Then $v_{-}: G \rightarrow \mathbf{C}$ satisfies the DPRC for wavenumber $k_{*}$ in $G$ if and only if $v_{+}: G^{\prime} \rightarrow \mathbf{C}$, given by $v_{+}(x)=v_{-}\left(x^{\prime}\right), x \in G^{\prime}$, satisfies the UPRC for wavenumber $k_{*}$ in $G^{\prime}$.

Lemma 2.1 (see [7, Theorem 2.1]). Given $H \in \mathbf{R}$ and $v: U_{H} \rightarrow \mathbf{C}$, the following statements are equivalent:

(i) $v \in C^{2}\left(U_{H}\right), v \in L_{\infty}\left(U_{H} \backslash U_{a}\right)$ for all $a>H, \Delta v+k_{+}^{2} v=0$ in $U_{H}$, and $v$ satisfies the UPRC for wavenumber $k_{+}$;

(ii) $v \in L_{\infty}\left(U_{H} \backslash U_{a}\right)$ for some $a>H$ and $v$ satisfies (2.4) for each $h>H$ with $\phi=\left.v\right|_{\Gamma_{h}} ;$

(iii) $v \in C^{2}\left(U_{H}\right), v \in L_{\infty}\left(U_{H} \backslash U_{a}\right)$ for all $a>H, \Delta v+k_{+}^{2} v=0$ in $U_{H}$, and for every $h>H$ and radiating solution in $U_{H}, w$, such that the restrictions of $w$ and $\partial_{2} w$ to $\Gamma_{h}$ are in $L_{1}(\mathbf{R})$, it holds that

$$
\int_{\Gamma_{h}}\left(v \frac{\partial w}{\partial n}-w \frac{\partial v}{\partial n}\right) d s=0
$$

From Lemma 2.1 and Remark 2.1 we can deduce corresponding characteristics of downward propagating solutions of the Helmholtz equation.

For convenience, we now state a local regularity estimate used throughout the paper.

Lemma 2.2 (see [18, Theorem 3.9, Lemma 4.1]). If for some $A>0$ and $x \in \mathbf{R}^{2}$ it holds that $v \in L_{\infty}\left(B_{A}(x)\right)$ and $\Delta v=f \in L_{\infty}\left(B_{A}(x)\right)$ (in a distributional sense), 
then $v \in C^{1}\left(B_{A}(x)\right)$ and

$$
|\nabla v(y)| \leq C A^{-1}\left(\|v\|_{\infty}+A^{2}\|f\|_{\infty}\right), \quad y \in B_{A / 2}(x),
$$

where $C$ is an absolute constant.

Remark 2.2. A consequence of Lemma 2.2 is that if $\Delta v+k^{2} v=0$ in some region $G$ and $v \in L_{\infty}(G), k \in L_{\infty}(G)$, then $v \in C^{1}(G)$ and $\nabla v$ is bounded in every compact subset of $G$. Further, if the sequence $\left(v_{n}\right) \subset L_{\infty}(G)$ is uniformly bounded, $\Delta v_{n}+$ $k_{*}^{2} v_{n}=0$ in $G$ for some $k_{*} \in \mathbf{C}$ and each $n$, and $v_{n}(x) \rightarrow v(x)$ uniformly on compact subsets of $G$, then $v \in C^{2}(G)$ and $\Delta v+k_{*}^{2} v=0$ in $G$.

Our problem of scattering of a time-harmonic plane wave by an inhomogeneous layer can now be formulated as the following boundary value problem.

Problem (P). Find $u \in C\left(\mathbf{R}^{2}\right)$ such that (i) $u$ satisfies the reduced wave equation (2.3) in a distributional sense; (ii) $u^{\mathrm{s}}$ and $u$ satisfy the UPRC and DPRC (2.4) and (2.5), respectively; and (iii) $u$ is bounded in $E_{-A}^{A}$ for every $A>0$.

Remark 2.3. From (iii) and Lemma 2.2, it follows that $u \in C^{1}\left(\mathbf{R}^{2}\right) \cap C^{2}\left(U_{B}\right) \cap$ $C^{2}\left(\mathbf{R}^{2} \backslash \bar{U}\right)$ and

$$
\sup _{x \in E_{-A}^{A}}[|\nabla u(x)|+|u(x)|]<\infty
$$

for every $A>0$. Further, by (2.3) and standard local regularity results [18], we have that $u \in H_{\mathrm{loc}}^{2}\left(\mathbf{R}^{2}\right)$.

Remark 2.4. The radiation conditions (2.4) and (2.5) are generalizations of the standard radiation conditions for one-dimensional periodic gratings. Precisely, it was proven in [4] that if $u^{\mathrm{s}}$ has the usual representation as a Rayleigh expansion [2, 19, 20] in some $U_{\tau}$, then it also satisfies (2.4) for all $h>\tau$ and thus satisfies the UPRC. As a consequence, any upward or horizontally propagating plane wave satisfies the UPRC and, by Remark 2.1, any downward or horizontally propagating plane wave satisfies the DPRC.

In what follows we are concerned with deriving an equivalent integral equation formulation of Problem (P) and with establishing unique solvability for Problem (P), employing integral equation methods.

3. An integral equation formulation. For $h \in \mathbf{R}$ let $y_{h}^{\prime}=\left(y_{1}, 2 h-y_{2}\right)$ be the image of $y$ in $\Gamma_{h}$ and define

$$
G_{h}^{ \pm}(x, y)=\Phi\left(x, y ; k_{ \pm}\right)-\Phi\left(x, y_{h}^{\prime} ; k_{ \pm}\right), \quad x, y \in \mathbf{R}^{2}, \quad x \neq y .
$$

Then $G_{h}^{ \pm}$is the Dirichlet Green's function for $\Delta+k_{ \pm}^{2}$ in the half-planes $U_{h}$ and $\mathbf{R}^{2} \backslash \bar{U}_{h}$. It follows from [6, Lemma 3.1] that, for some constant $C$ depending only on $k_{ \pm}$and $h$,

$$
\left|G_{h}^{ \pm}(x, y)\right|, \quad\left|\nabla_{x} G_{h}^{ \pm}(x, y)\right|, \quad\left|\nabla_{y} G_{h}^{ \pm}(x, y)\right| \leq C \frac{\left(1+x_{2}\right)\left(1+y_{2}\right)}{|x-y|^{3 / 2}}
$$

if $x, y \in U_{h}$ or $x, y \in \mathbf{R}^{2} \backslash \bar{U}_{h}$ with $|x-y| \geq 1$. On the other hand, from asymptotic properties of the Hankel function it follows that

$$
\left|G_{h}^{ \pm}(x, y)\right| \leq C(1+|\log | x-y||), \quad\left|\nabla_{x} G_{h}^{ \pm}(x, y)\right|, \quad\left|\nabla_{y} G_{h}^{ \pm}(x, y)\right| \leq C|x-y|^{-1}
$$

if $x, y \in U_{h}$ or $x, y \in \mathbf{R}^{2} \backslash \bar{U}_{h}$ with $|x-y| \leq 1$. It follows from (3.1) and (3.2) that, for $0 \leq h \leq B$

$$
\left|G_{h}^{ \pm}(x, y)\right|, \quad\left|\nabla_{x} G_{h}^{ \pm}(x, y)\right|, \quad\left|\nabla_{y} G_{h}^{ \pm}(x, y)\right| \leq C_{b}\left(1+\left|x_{1}-y_{1}\right|\right)^{-3 / 2}
$$


if $y \in \bar{E}_{h}^{B}, x \in \Gamma_{b}$, with $b>B$, or if $y \in \bar{E}_{0}^{h}, x \in \Gamma_{b}$, with $b<0$, where $C_{b}$ depends only on $b, B, h$, and $k_{ \pm}$.

Let $u^{\mathrm{r}}$ denote the upward propagating plane wave $u^{\mathrm{r}}(x)=-\exp \left(i k_{+} x_{c}^{\prime} \cdot \alpha\right)$, $x \in \mathbf{R}^{2}$.

Theorem 3.1. Let $u$ be a solution of Problem (P), and let $0 \leq c<d \leq B$. Then we have

$$
\begin{gathered}
u(x)=u^{\mathrm{i}}(x)+u^{\mathrm{r}}(x)+\int_{E_{c}^{B}} u(y)\left[k^{2}(y)-k_{+}^{2}\right] G_{c}^{+}(x, y) d y \\
\quad+\int_{\Gamma_{c}} u(y) \frac{\partial G_{c}^{+}(x, y)}{\partial y_{2}} d s(y), \quad x \in U_{c}, \\
u(x)=\int_{E_{0}^{d}} u(y)\left[k^{2}(y)-k_{-}^{2}\right] G_{d}^{-}(x, y) d y \\
\quad-\int_{\Gamma_{d}} u(y) \frac{\partial G_{d}^{-}(x, y)}{\partial y_{2}} d s(y), \quad x \in \mathbf{R}^{2} \backslash \bar{U}_{d} .
\end{gathered}
$$

Remark 3.1. In view of (3.1) and (3.2), (A1), and the fact that $u \in B C\left(\overline{E_{B}}\right)$, the integrals in (3.4) and (3.5) are well defined.

Proof. Take $x \in U_{c}$, choose $b>\max \left(x_{2}, B\right), A>\left|x_{1}\right|$, and $\epsilon>0$ sufficiently small and apply Green's second theorem to $G_{c}^{+}(x, \cdot)$ and $u$ in the bounded region $E_{c}^{b}(A) \backslash \overline{B_{\epsilon}(x)}$, and then let $\epsilon \rightarrow 0$ to obtain that

$$
\begin{aligned}
u(x)= & \int_{E_{c}^{B}(A)} u(y)\left[k^{2}(y)-k_{+}^{2}\right] G_{c}^{+}(x, y) d y \\
& \quad+\int_{\partial\left(E_{c}^{b}(A)\right)}\left[G_{c}^{+}(x, y) \frac{\partial u}{\partial n}(y)-u(y) \frac{\partial G_{c}^{+}(x, y)}{\partial n(y)}\right] d s(y) .
\end{aligned}
$$

Letting $A \rightarrow \infty$ in (3.6), in view of (3.1), we find that

$$
u(x)=\int_{E_{c}^{B}} u(y)\left[k^{2}(y)-k_{+}^{2}\right] G_{c}^{+}(x, y) d y+\int_{\Gamma_{c}} u(y) \frac{\partial G_{c}^{+}(x, y)}{\partial y_{2}} d s(y)+I_{b},
$$

where

$$
I_{b}=\int_{\Gamma_{b}}\left[G_{c}^{+}(x, y) \frac{\partial u}{\partial n}(y)-u(y) \frac{\partial G_{c}^{+}(x, y)}{\partial n(y)}\right] d s(y) .
$$

Now $v=u^{\mathrm{i}}+u^{\mathrm{r}}$ satisfies the Helmholtz equation $\Delta v+k_{+}^{2} v=0$ in $U_{c}$ and the Dirichlet condition $v=0$ on $\Gamma_{c}$, so that by the same argument used to derive (3.7), we can show that $v(x)=\tilde{I}_{b}$, where $\tilde{I}_{b}$ is given by (3.8) but with $u$ replaced by $v$. It follows that

$$
I_{b}=u^{\mathrm{i}}(x)+u^{\mathrm{r}}(x)+\int_{\Gamma_{b}}\left[G_{c}^{+}(x, y) \frac{\partial w}{\partial n}(y)-w(y) \frac{\partial G_{c}^{+}(x, y)}{\partial n(y)}\right] d s(y),
$$

where $w=u^{\mathrm{s}}-u^{\mathrm{r}}$. Further, by Remark 2.4, $u^{\mathrm{r}}$ and thus $w$ satisfies the UPRC. Also, $G_{c}^{+}(x, \cdot)$ is a radiating solution in $U_{\tau}$ for $\tau>\max \left(x_{2}, B\right)$ so that, in view of (3.1) and 
the equivalence of (i) and (iii) in Lemma 2.1, the integral in (3.9) vanishes. Thus (3.4) follows.

Take $x \in \mathbf{R}^{2} \backslash \bar{U}_{d}$, and choose $a<\min \left(x_{2}, 0\right), A>\left|x_{1}\right|$, and $\epsilon>0$ sufficiently small. Then (3.5) can be derived similarly by applying Green's second theorem to $G_{d}^{-}(x, \cdot)$ and $u$ in the bounded region $E_{a}^{d}(A) \backslash \overline{B_{\epsilon}(x)}$, letting $\epsilon \rightarrow 0, A \rightarrow \infty$, and finally utilizing the equivalence of (i) and (iii) in Lemma 2.1 and noting Remark 2.1.

The next two lemmas state properties of volume and surface potentials of the type appearing in (3.4) and (3.5). Lemma 3.1(i) was proved as Lemma 3.1 in [7] while Lemma 3.2(i) was proved as Theorem 3.2 in [5]. In both lemmas the assertion (ii) is a consequence of (i) on noting Remark 2.1.

Lemma 3.1. (i) Define the volume potential $v_{+}$with density $\phi_{+} \in L_{\infty}\left(E_{c}^{B}\right)$ by

$$
v_{+}(x)=\int_{E_{c}^{B}} G_{c}^{+}(x, y) \phi_{+}(y) d y, \quad x \in \bar{U}_{c},
$$

and extend the definition of $\phi_{+}$to $U_{c}$ by setting $\phi_{+}(x)=0, x \in U_{B}$. Then $v_{+} \in$ $C^{1}\left(\bar{U}_{c}\right) \cap L_{\infty}\left(E_{c}^{b}\right)$ for $b>c, v_{+}=0$ on $\Gamma_{c}, \Delta v_{+}+k_{+}^{2} v_{+}=-\phi_{+}$in $U_{c}$, and $v_{+}$ satisfies the UPRC.

(ii) Define the volume potential $v_{-}$with density $\phi_{-} \in L_{\infty}\left(E_{0}^{d}\right)$ by

$$
v_{-}(x)=\int_{E_{0}^{d}} G_{d}^{-}(x, y) \phi_{-}(y) d y, \quad x \in \mathbf{R}^{2} \backslash U_{d},
$$

and extend the definition of $\phi_{-}$to $\mathbf{R}^{2} \backslash \bar{U}_{d}$ by setting $\phi_{-}(x)=0, x \in \mathbf{R}^{2} \backslash U$. Then $v_{-} \in C^{1}\left(\mathbf{R}^{2} \backslash U_{d}\right) \cap L_{\infty}\left(E_{a}^{d}\right)$ for $a<d, v_{-}=0$ on $\Gamma_{d}, \Delta v_{-}+k_{-}^{2} v_{-}=-\phi_{-}$in $\mathbf{R}^{2} \backslash \bar{U}_{d}$, and $v_{-}$satisfies the DPRC.

Lemma 3.2. (i) Define the double layer potential $D_{+}$with density $\psi_{+} \in B C\left(\Gamma_{c}\right)$ by

$$
D_{+}(x)=\int_{\Gamma_{c}} \frac{\partial G_{c}^{+}(x, y)}{\partial y_{2}} \psi_{+}(y) d s(y), \quad x \in U_{c} .
$$

Then $D_{+} \in C\left(\bar{U}_{c}\right) \cap C^{2}\left(U_{c}\right) \cap L_{\infty}\left(E_{c}^{b}\right)$ for $b>c, D_{+}=\psi_{+}$on $\Gamma_{c}, \Delta D_{+}+k_{+}^{2} D_{+}=0$ in $U_{c}$, and $D_{+}$satisfies the UPRC.

(ii) Define the double layer potential $D_{-}$with density $\psi_{-} \in B C\left(\Gamma_{d}\right)$ by

$$
D_{-}(x)=\int_{\Gamma_{d}} \frac{\partial G_{d}^{-}(x, y)}{\partial y_{2}} \psi_{-}(y) d s(y), \quad x \in \mathbf{R}^{2} \backslash \bar{U}_{d} .
$$

Then $D_{-} \in C\left(\mathbf{R}^{2} \backslash U_{d}\right) \cap C^{2}\left(\mathbf{R}^{2} \backslash \bar{U}_{d}\right) \cap L_{\infty}\left(E_{a}^{d}\right)$ for $a<d, D_{-}=-\psi_{-}$on $\Gamma_{d}, \Delta D_{-}+$ $k_{-}^{2} D_{-}=0$ in $\mathbf{R}^{2} \backslash \bar{U}_{d}$, and $D_{-}$satisfies the DPRC.

As in Theorem 3.1, choose $c$ and $d$ so that $0 \leq c<d \leq B$ and let $\lambda=\left(k_{+} c+\right.$ $\left.k_{-} d\right) /\left(k_{-}+k_{+}\right)$so that $k_{-}(d-\lambda)=k_{+}(\lambda-c)$. Suppose that $u$ satisfies Problem (P) and let $\psi_{1}=\left.u\right|_{E_{\lambda}^{B}}, \psi_{2}=\left.u\right|_{E_{0}^{\lambda}}$, and $k^{ \pm}=k^{2}-k_{ \pm}^{2}$. Then, by Theorem 3.1, $\psi_{1} \in B C\left(\bar{E}_{\lambda}^{B}\right)$ and $\psi_{2} \in B C\left(\bar{E}_{0}^{\lambda}\right)$ satisfy the pair of second-kind integral equations

$$
\psi_{1}(x)=u^{i}(x)+u^{r}(x)+\int_{E_{\lambda}^{B}} \psi_{1}(y) k^{+}(y) G_{c}^{+}(x, y) d y+\int_{E_{c}^{\lambda}} \psi_{2}(y) k^{+}(y) G_{c}^{+}(x, y) d y
$$




$$
\begin{aligned}
& +\int_{\Gamma_{c}} \psi_{2}(y) \frac{\partial G_{c}^{+}(x, y)}{\partial y_{2}} d s(y), \quad x \in \bar{E}_{\lambda}^{B}, \\
\psi_{2}(x)= & \int_{E_{0}^{\lambda}} \psi_{2}(y) k^{-}(y) G_{d}^{-}(x, y) d y+\int_{E_{\lambda}^{d}} \psi_{1}(y) k^{-}(y) G_{d}^{-}(x, y) d y
\end{aligned}
$$

$$
-\int_{\Gamma_{d}} \psi_{1}(y) \frac{\partial G_{d}^{-}(x, y)}{\partial y_{2}} d s(y), \quad x \in \bar{E}_{0}^{\lambda} .
$$

Conversely, suppose now that $\psi_{1} \in B C\left(\bar{E}_{\lambda}^{B}\right)$ and $\psi_{2} \in B C\left(\bar{E}_{0}^{\lambda}\right)$ satisfy the integral equations (3.10) and (3.11) and define $u$ as follows:

$$
\begin{aligned}
u(x)= & u^{\mathrm{i}}(x)+u^{\mathrm{r}}(x)+\int_{E_{\lambda}^{B}} \psi_{1}(y) k^{+}(y) G_{c}^{+}(x, y) d y+\int_{E_{c}^{\lambda}} \psi_{2}(y) k^{+}(y) G_{c}^{+}(x, y) d y \\
& +\int_{\Gamma_{c}} \psi_{2}(y) \frac{\partial G_{c}^{+}(x, y)}{\partial y_{2}} d s(y), \quad x \in U_{c}, \\
u(x)= & \lim _{y \rightarrow x, y \in U_{c}} u(y), \quad x \in \Gamma_{c}, \\
u(x)= & \int_{E_{0}^{\lambda}} \psi_{2}(y) k^{-}(y) G_{d}^{-}(x, y) d y+\int_{E_{\lambda}^{d}} \psi_{1}(y) k^{-}(y) G_{d}^{-}(x, y) d y \\
& -\int_{\Gamma_{d}} \psi_{1}(y) \frac{\partial G_{d}^{-}(x, y)}{\partial y_{2}} d s(y), \quad x \in \mathbf{R}^{2} \backslash \bar{U}_{c} .
\end{aligned}
$$

Then it follows, provided $d-c$ is small enough, that $u$ is a solution of Problem (P). To see this define $v$ by

$$
\begin{aligned}
v(x)= & \int_{E_{0}^{\lambda}} \psi_{2}(y) k^{-}(y) G_{d}^{-}(x, y) d y+\int_{E_{\lambda}^{d}} \psi_{1}(y) k^{-}(y) G_{d}^{-}(x, y) d y \\
& -\int_{\Gamma_{d}} \psi_{1}(y) \frac{\partial G_{d}^{-}(x, y)}{\partial y_{2}} d s(y), \quad x \in \mathbf{R}^{2} \backslash \bar{U}_{d}, \\
v(x)= & \lim _{y \rightarrow x, y \in \mathbf{R}^{2} \backslash \bar{U}_{d}} v(y), \quad x \in \Gamma_{d} .
\end{aligned}
$$

Then, comparing (3.10) and (3.12), $\psi_{1}=\left.u\right|_{\bar{E}_{\lambda}^{B}}$, and comparing (3.11) and (3.15), $\psi_{2}=\left.v\right|_{E_{0}^{\lambda}}$. Also, by Lemmas 3.1 and 3.2 applied to (3.12) and (3.15), $\psi_{2}=u$ on $\Gamma_{c}$ and $\psi_{1}=v$ on $\Gamma_{d}$. Thus $u=v$ on $\Gamma_{c}$ and $\Gamma_{d}$. Define $w=u-v$. Then it is clear from Lemmas 3.1 and 3.2 again, together with the above results, that (i) $w$ is bounded in $E_{c}^{d}$ and $w \in C\left(\bar{E}_{c}^{d}\right) \cap C^{1}\left(E_{c}^{d}\right)$; (ii) $\Delta w+\hat{k}^{2} w=0$ in $E_{c}^{d}$, where $\hat{k}(x)=k_{-}, x \in E_{\lambda}^{d},=k_{+}$, $x \in E_{c}^{\lambda}$; (iii) $w=0$ on $\Gamma_{c}$ and $\Gamma_{d}$. Now, consider the following eigenvalue problem: find $z \in C^{1}[c, d] \cap H^{2}(c, d), \Lambda \in \mathbf{R}$, such that $-z^{\prime \prime}-q z=\Lambda z$ in $(c, d)$ and $z(c)=z(d)=0$, where $q\left(x_{2}\right)=k_{-}, \lambda<x_{2}<d,=k_{+}, c<x_{2}<\lambda$. Provided this problem has only positive eigenvalues $\Lambda>0$, and this is the case if $(d-\lambda) k_{-}=(\lambda-c) k_{+}<\pi / 2$, i.e., provided $2 k_{+} k_{-}(d-c)<\pi\left(k_{+}+k_{-}\right)$, then an elementary separation of variables argument establishes that $w \equiv 0$ in $E_{c}^{d}$ and hence $u \equiv v$ in $E_{c}^{d}$. It is now easy to see, by 
further applications of Lemmas 3.1 and 3.2, that $u$, defined by (3.12)-(3.14), satisfies Problem (P). Thus we have the following equivalence theorem between Problem (P) and the integral equation problem (3.10) and (3.11).

Theorem 3.2. If $u \in C\left(\mathbf{R}^{2}\right)$ is a solution of Problem $(\mathrm{P})$, then $\psi_{1}:=\left.u\right|_{\bar{E}_{\lambda}^{B}}$ and $\psi_{2}:=\left.u\right|_{\bar{E}_{0}^{\lambda}}$ satisfy the integral equations (3.10) and (3.11). Conversely, suppose that $\psi_{1} \in B C\left(\bar{E}_{\lambda}^{B}\right)$ and $\psi_{2} \in B C\left(\bar{E}_{0}^{\lambda}\right)$ satisfy the integral equations (3.10) and (3.11) and define $u$ by (3.12)-(3.14). Then, provided $(d-\lambda) k_{-}=(\lambda-c) k_{+}$and $2 k_{+} k_{-}(d-c)<$ $\pi\left(k_{+}+k_{-}\right)$, u satisfies Problem $(\mathrm{P})$.

Remark 3.2. Let $(d-\lambda) k_{-}=(\lambda-c) k_{+}$and $2 k_{+} k_{-}(d-c)<\pi\left(k_{+}+k_{-}\right)$. Then from Theorem 3.2 it follows that in order to prove the existence of a solution to Problem $(\mathrm{P})$, it is enough to show that the pair of integral equations (3.10) and (3.11) has a solution. This will be done in section 6 .

4. A basic inequality. In this section a basic inequality satisfied by solutions of (2.3) is established, which is a key step in the proof of the uniqueness theorem.

Suppose that $u \in C\left(\mathbf{R}^{2}\right)$ satisfies (2.3). Then, by Remark 2.3, $u \in C^{1}\left(\mathbf{R}^{2}\right) \cap$ $H_{\text {loc }}^{2}\left(\mathbf{R}^{2}\right)$. Let $\eta<c<d<\rho$ with $\eta, \rho$ being as defined in assumptions (A3) or (A5) and define, for $t \in \mathbf{R}$ and $A>0$,

$$
\begin{aligned}
J_{A}(t)= & \Im \int_{\Gamma_{t}(A)} \bar{u} \partial_{2} u d s, \quad L_{A}(t)=\Re \int_{\Gamma_{t}(A)} \bar{u} \partial_{2} u d s, \\
I_{A}^{ \pm}(t)= & \int_{\Gamma_{t}(A)}\left\{\left|\partial_{2} u\right|^{2}-\left|\partial_{1} u\right|^{2}+k_{ \pm}^{2}|u|^{2}\right\} d s, \\
K_{A}= & \int_{E_{\eta}^{B}(A)}|u|^{2}\left|k^{2}-k_{+}^{2}\right| d x+\int_{E_{0}^{\rho}(A)}|u|^{2}\left|k^{2}-k_{-}^{2}\right| d x \\
& +\int_{\Gamma_{c}(A)}|u|^{2} d s+\int_{\Gamma_{d}(A)}|u|^{2} d s .
\end{aligned}
$$

Let $a<0<B<b$, and for $t \in \mathbf{R}$, let $\gamma(t)=\left\{\left(t, x_{2}\right) \mid a \leq x_{2} \leq b\right\}$.

ThEOREM 4.1. Assume that (A3) holds or that both (A4) and (A5) hold. Then, for some nonnegative constants $C_{j}, j=1,2,3$, there holds

$$
\begin{aligned}
K_{A} \leq & C_{1}\left[(b-\beta) I_{A}^{+}(b)-(a-\beta) I_{A}^{-}(a)\right]+C_{1}\left[L_{A}(b)-L_{A}(a)\right] \\
& +C_{2}\left[J_{A}(a)-J_{A}(b)\right]+C_{3} R_{1}(A)+C_{1} R_{2}(A),
\end{aligned}
$$

for all $A>0$, where

$$
R_{1}(A)=\left[\int_{\gamma(A)}+\int_{\gamma(-A)}\right]\left|\bar{u} \partial_{1} u\right| d s
$$

and

$$
R_{2}(A)=\Re\left[\int_{\gamma(A)}-\int_{\gamma(-A)}\right]\left[2\left(x_{2}-\beta\right) \partial_{2} \bar{u} \partial_{1} u+\bar{u} \partial_{1} u\right] d s .
$$

Proof. First we will deduce the inequality (4.5) in the case that (A3) holds. 
Apply Green's first theorem to $u$ and $\bar{u}$ in $E_{a}^{b}(A)$ and take the imaginary part of the result thus obtained to get that since $\Im\left(k^{2}(x)\right)=0$ for $x_{2}>B$ and $x_{2}<0$,

$$
\int_{E_{B}(A)} \Im\left(k^{2}\right)|u|^{2} d x+J_{A}(b)-J_{A}(a) \leq R_{1}(A) .
$$

Let $\theta \in C^{2}(\mathbf{R})$ be such that $0 \leq \theta(t) \leq 1$ for $t \in \mathbf{R}, \theta(t)=1$ for $c \leq t \leq d$, and $\theta(t)=0$ for $t \geq \rho$ and $t \leq \eta$. Then, by applying Green's first theorem to $u$ and $\theta\left(x_{2}\right) \bar{u}$ in $E_{B}(A)$ and taking the real part of the result thus obtained, we obtain on integrating by parts that

$$
\int_{E_{B}(A)} \theta\left(x_{2}\right)|\nabla u|^{2} d x \leq \int_{E_{B}(A)}\left[\Re\left(k^{2}\right) \theta\left(x_{2}\right)+\frac{1}{2} \theta^{\prime \prime}\left(x_{2}\right)\right]|u|^{2} d x+R_{1}(A),
$$

which together with the definition of $\theta$ implies that

$$
\int_{E_{c}^{d}(A)}|\nabla u|^{2} d x \leq\left(\|k\|_{\infty}^{2}+C\right) \int_{E_{\eta}^{\rho}(A)}|u|^{2} d x+R_{1}(A),
$$

for some constant $C>0$ depending only on the choice of $\theta$.

Now, for any $r, t \in \mathbf{R}$,

$$
u\left(\left(x_{1}, r\right)\right)-u\left(\left(x_{1}, t\right)\right)=\int_{t}^{r} \partial_{2} u(x) d x_{2}, \quad x_{1} \in \mathbf{R},
$$

so that using the Cauchy-Schwarz inequality,

$$
\left|u\left(\left(x_{1}, t\right)\right)\right|^{2} \leq 2\left|u\left(\left(x_{1}, r\right)\right)\right|^{2}+2(r-t) \int_{t}^{r}\left|\partial_{2} u(x)\right|^{2} d x_{2}, \quad x_{1} \in \mathbf{R} .
$$

From (4.9) it follows that, for $R<T, r, t \in[R, T]$,

$$
\int_{\Gamma_{t}(A)}|u|^{2} d s \leq 2 \int_{\Gamma_{r}(A)}|u|^{2} d s+2(T-R) \int_{E_{R}^{T}(A)}\left|\partial_{2} u\right|^{2} d x
$$

and hence that

$$
(T-R) \int_{\Gamma_{t}(A)}|u|^{2} d s \leq 2 \int_{E_{R}^{T}(A)}|u|^{2} d x+2(T-R)^{2} \int_{E_{R}^{T}(A)}\left|\partial_{2} u\right|^{2} d x .
$$

Thus, assuming that (A3) holds, the required inequality (4.5), with $C_{1}=0$, follows from (4.6), (4.7), and (4.11) with $R=c, T=d, t=c, d$.

Suppose now that (A4) and (A5) hold. Multiplying (2.3) by $2\left(x_{2}-\beta\right) \partial_{2} \bar{u}+\bar{u}$, integrating over $E_{a}^{b}(A)$, and taking the real part, we obtain on noting that $\Im\left(k^{2}(x)\right)=$ 0 for $x_{2}>B$ and $x_{2}<0$,

$2 \int_{E_{a}^{b}(A)}\left|\partial_{2} u\right|^{2} d x=\Re \int_{E_{a}^{b}(A)}\left\{2 \nabla \cdot\left[\left(x_{2}-\beta\right) \partial_{2} \bar{u} \nabla u\right]-\partial_{2}\left[\left(x_{2}-\beta\right)|\nabla u|^{2}\right]+\nabla \cdot(\bar{u} \nabla u)\right\} d x$ 


$$
\begin{aligned}
& +\int_{E_{a}^{b}(A)} \Re\left(k^{2}\right) \partial_{2}\left[\left(x_{2}-\beta\right)|u|^{2}\right] d x+2 \int_{E_{B}(A)}\left(x_{2}-\beta\right) \Im\left(k^{2}\right) \Im\left\{\bar{u} \partial_{2} u\right\} d x \\
= & (b-\beta) \int_{\Gamma_{b}(A)}\left(\left|\partial_{2} u\right|^{2}-\left|\partial_{1} u\right|^{2}\right) d s-(a-\beta) \int_{\Gamma_{a}(A)}\left(\left|\partial_{2} u\right|^{2}-\left|\partial_{1} u\right|^{2}\right) d s \\
& +L_{A}(b)-L_{A}(a)+R_{2}(A)+\int_{E_{a}^{b}(A)} \Re\left(k^{2}\right) \partial_{2}\left[\left(x_{2}-\beta\right)|u|^{2}\right] d x \\
& +2 \int_{E_{B}(A)}\left(x_{2}-\beta\right) \Im\left(k^{2}\right) \Im\left\{\bar{u} \partial_{2} u\right\} d x .
\end{aligned}
$$

Now, if $\Re\left(k^{2}\right) \in C^{1}\left(\mathbf{R}^{2}\right)$, then from (A4) we have that $\left(x_{2}-\beta\right) \partial_{2}\left(\Re\left(k^{2}\right)\right) \geq 0$, and integrating by parts, we obtain that

$$
\begin{aligned}
\int_{E_{a}^{b}(A)} \Re\left(k^{2}\right) \partial_{2}\left[\left(x_{2}-\beta\right)|u|^{2}\right] d x & \leq(b-\beta) k_{+}^{2} \int_{\Gamma_{b}(A)}|u|^{2} d s-(a-\beta) k_{-}^{2} \int_{\Gamma_{a}(A)}|u|^{2} d s \\
& =\int_{E_{a}^{b}(A)} \tilde{k}^{2} \partial_{2}\left[\left(x_{2}-\beta\right)|u|^{2}\right] d x .
\end{aligned}
$$

Thus

$$
G_{A} \equiv \int_{E_{a}^{b}(A)}\left[\tilde{k}^{2}-\Re\left(k^{2}\right)\right] \partial_{2}\left[\left(x_{2}-\beta\right)|u|^{2}\right] d x \geq 0 .
$$

In the general case where $k \in L_{\infty}\left(\mathbf{R}^{2}\right)$, let $\phi(x)=\Re\left(k^{2}(x)\right), \psi(x)=\left(x_{2}-\beta\right)|u(x)|^{2}$, and for $h \in \mathbf{R}$, let $\phi_{h}(x)=\phi\left(x+h e_{2}\right), \psi_{h}(x)=\psi\left(x+h e_{2}\right)$. Then, since $\phi\left(\psi_{h}-\psi\right)+$ $\phi_{h}\left(\psi_{h}-\psi\right)=2\left(\phi_{h} \psi_{h}-\phi \psi\right)-\left(\phi_{h}-\phi\right)\left(\psi+\psi_{h}\right)$, we have that for sufficiently small $h>0$,

$$
\begin{aligned}
& \int_{E_{a}^{b}(A)} \phi\left(\psi_{h}-\psi\right) d x+\int_{E_{a+h}^{b+h}(A)} \phi\left(\psi-\psi_{-h}\right) d x \\
& =2 \int_{E_{b}^{b+h}(A)} \phi \psi d x-2 \int_{E_{a}^{a+h}(A)} \phi \psi d x-\int_{E_{a}^{b}(A)}\left(\phi_{h}-\phi\right)\left(\psi+\psi_{h}\right) d x .
\end{aligned}
$$

By using (A4) the last term on the right-hand side of (4.15) can be estimated as follows. First, in the cases where $\beta \leq a$ and $\beta \geq b+h$, it is easy to see that

$$
I \equiv-\int_{E_{a}^{b}(A)}\left(\phi_{h}-\phi\right)\left(\psi+\psi_{h}\right) d x \leq 0,
$$

while if $a<\beta<b+h$, then

$$
I \leq 2\|k\|_{\infty}^{2} \int_{E_{\beta-h}^{\beta}(A)}\left|\psi+\psi_{h}\right| d x \equiv I_{h} .
$$


Therefore, $I \leq I_{h}$ in any case, and thus it follows from (4.15) that

$$
\begin{gathered}
\int_{E_{a}^{b}(A)} \phi\left(\psi_{h}-\psi\right) d x+\int_{E_{a+h}^{b+h}(A)} \phi\left(\psi-\psi_{-h}\right) d x \\
\leq 2 k_{+}^{2} \int_{E_{b}^{b+h}(A)} \psi d x-2 k_{-}^{2} \int_{E_{a}^{a+h}(A)} \psi d x+I_{h}
\end{gathered}
$$

on using (A2). Since $\psi \in C^{1}\left(\mathbf{R}^{2}\right)$ and $\psi=0$ on $\Gamma_{\beta}$, dividing (4.16) by $2 h$ and taking the limit $h \rightarrow 0$ we obtain that (4.13) and (4.14) hold in the general case.

It follows from (4.12) that

$$
\begin{aligned}
2 \int_{E_{a}^{b}(A)}\left|\partial_{2} u\right|^{2} d x+G_{A}= & (b-\beta) I_{A}^{+}(b)-(a-\beta) I_{A}^{-}(a)+L_{A}(b)-L_{A}(a)+R_{2}(A) \\
& +2 \int_{E_{B}(A)}\left(x_{2}-\beta\right) \Im\left(k^{2}\right) \Im\left\{\bar{u} \partial_{2} u\right\} d x .
\end{aligned}
$$

Since $0 \leq \Im\left(k^{2}\right) \leq\|k\|_{\infty}^{2}$, the Cauchy-Schwarz inequality yields that

$$
2 \int_{E_{B}(A)}\left(x_{2}-\beta\right) \Im\left(k^{2}\right) \Im\left\{\bar{u} \partial_{2} u\right\} d x \leq \int_{E_{B}(A)}\left|\partial_{2} u\right|^{2} d x+(B+|\beta|)^{2}\|k\|_{\infty}^{2} \int_{E_{B}(A)} \Im\left(k^{2}\right)|u|^{2} d x .
$$

Thus, it follows from (4.17), (4.18), and (4.6) that

$$
\begin{aligned}
\int_{E_{a}^{b}(A)}\left|\partial_{2} u\right|^{2} d x+G_{A} \leq & (b-\beta) I_{A}^{+}(b)-(a-\beta) I_{A}^{-}(a)+L_{A}(b)-L_{A}(a)+R_{2}(A) \\
& +(B+|\beta|)^{2}\|k\|_{\infty}^{2}\left[J_{A}(a)-J_{A}(b)+R_{1}(A)\right] \equiv F_{A} .
\end{aligned}
$$

Now, from (4.19) and the fact that $G_{A} \geq 0$, it is seen that

$$
\int_{E_{a}^{b}(A)}\left|\partial_{2} u\right|^{2} d x \leq F_{A}
$$

On the other hand, since $2\left|\left(x_{2}-\beta\right) \Re\left(\bar{u} \partial_{2} u\right)\right| \leq|u|^{2} / 2+2(b+|\beta|)^{2}\left|\partial_{2} u\right|^{2}$ in $E_{a}^{b}$, we have

$$
\partial_{2}\left[\left(x_{2}-\beta\right)|u|^{2}\right]=|u|^{2}+2\left(x_{2}-\beta\right) \Re\left(\bar{u} \partial_{2} u\right) \geq|u|^{2} / 2-2(b+|\beta|)^{2}\left|\partial_{2} u\right|^{2},
$$

for $x \in E_{a}^{b}$, so that on noting that $\tilde{k}^{2} \geq \Re\left(k^{2}\right)$ by (A4),

$$
G_{A} \geq \frac{1}{2} \int_{E_{a}^{b}(A)}\left[\tilde{k}^{2}-\Re\left(k^{2}\right)\right]|u|^{2} d x-4(b+|\beta|)^{2}|| k \|_{\infty}^{2} \int_{E_{a}^{b}(A)}\left|\partial_{2} u\right|^{2} d x .
$$

This, together with (4.19) and (4.20), implies that

$$
\int_{E_{a}^{b}(A)}\left[\tilde{k}^{2}-\Re\left(k^{2}\right)\right]|u|^{2} d x \leq 2\left[1+4(b+|\beta|)^{2}\|k\|_{\infty}^{2}\right] F_{A} .
$$


We now make use of (4.20) and (4.21) to derive the required inequality (4.5). First, using (4.9) and the fact that $\Re\left(k^{2}\right) \leq \tilde{k}^{2}$ by (A4), we obtain (cf. (4.11)) that

$$
\begin{aligned}
& \int_{E_{c}^{d}(A)}\left[\tilde{k}^{2}-\Re\left(k^{2}\right)\right]\left|u\left(\left(x_{1}, c\right)\right)\right|^{2} d x \\
& \quad \leq 2 \int_{E_{c}^{d}(A)}\left[\tilde{k}^{2}-\Re\left(k^{2}\right)\right]|u|^{2} d x+4(d-c)^{2}\|k\|_{\infty}^{2} \int_{E_{c}^{d}(A)}\left|\partial_{2} u\right|^{2} d x .
\end{aligned}
$$

Using (A4) and (A5) yields that

$$
(d-c) \lambda_{3} \int_{\Gamma_{c}(A)}|u|^{2} d s \leq 2 \int_{E_{a}^{b}(A)}\left[\tilde{k}^{2}-\Re\left(k^{2}\right)\right]|u|^{2} d x+4 B^{2}\|k\|_{\infty}^{2} \int_{E_{a}^{b}(A)}\left|\partial_{2} u\right|^{2} d x .
$$

From (4.10) with $R=a, T=b, r=c$, we obtain that

$$
\int_{E_{a}^{b}(A)}|u|^{2} d x \leq 2(b-a) \int_{\Gamma_{c}(A)}|u|^{2} d s+2(b-a)^{2} \int_{E_{a}^{b}(A)}\left|\partial_{2} u\right|^{2} d x .
$$

Thus, utilizing (4.20) and (4.21) together with (4.23), (4.24), and (4.11), with $R=a$, $T=b, t=d$, it follows that $K_{A}$ is bounded by a multiple of $F_{A}$. Thus the required result (4.5) holds with $C_{2}=C_{3}$.

5. Uniqueness of solution. In this section we establish the following uniqueness theorem for Problem $(\mathrm{P})$.

THEOREM 5.1. If (A3) holds or both (A4) and (A5) hold, then Problem (P) has at most one solution.

We prove this theorem by showing that the homogeneous version of Problem (P) has only the trivial solution. Since guided waves are solutions of the homogeneous problem (see Definition A.1 and Theorem A.1 in the appendix), we have immediately the following corollary.

COROllary 5.1. If (A3) holds or both (A4) and (A5) hold, then there are no guided wave solutions to the homogeneous problem.

In the proof of Theorem 5.1 we utilize the following two lemmas, the first of which is a special case of Lemma $\mathrm{A}$ in [8].

Lemma 5.1. Suppose that $F \in L_{\infty}(\mathbf{R})$ and that, for some nonnegative constants $C, \epsilon$, and $A_{0}$,

$\int_{-A}^{A}|F(t)|^{2} d t \leq C \int_{\mathbf{R} \backslash[-A, A]} G_{A}^{2}(t) d t+C \int_{-A}^{A}\left(G_{\infty}(t)-G_{A}(t)\right) G_{\infty}(t) d t+\epsilon, \quad A>A_{0}$, where, for $A_{0}<A \leq+\infty$,

$$
G_{A}(s)=\int_{-A}^{A}(1+|s-t|)^{-3 / 2}|F(t)| d t, \quad s \in \mathbf{R} .
$$

Then $F \in L_{2}(\mathbf{R})$ and

$$
\int_{-\infty}^{+\infty}|F(t)|^{2} d t \leq \epsilon
$$


Lemma 5.2. If $\phi_{+} \in L_{2}\left(\Gamma_{H}\right) \cap L_{\infty}\left(\Gamma_{H}\right), \phi_{-} \in L_{2}\left(\Gamma_{h}\right) \cap L_{\infty}\left(\Gamma_{h}\right)$, and $v_{ \pm}$are defined by (2.4) and (2.5), respectively, then the restrictions of $v_{+}, \partial_{1} v_{+}$, and $\partial_{2} v_{+}$to $\Gamma_{b}$ are in $L_{2}\left(\Gamma_{b}\right) \cap B C\left(\Gamma_{b}\right)$ for $b>H$; the restrictions of $v_{-}, \partial_{1} v_{-}$, and $\partial_{2} v_{-}$to $\Gamma_{a}$ are in $L_{2}\left(\Gamma_{a}\right) \cap B C\left(\Gamma_{a}\right)$ for $a<h$; and

$$
\begin{aligned}
& \Im \int_{\Gamma_{b}} \bar{v}_{+} \partial_{2} v_{+} d s \geq 0, \quad \Re \int_{\Gamma_{b}} \bar{v}_{+} \partial_{2} v_{+} d s \leq 0, \\
& \int_{\Gamma_{b}}\left[\left|\partial_{2} v_{+}\right|^{2}-\left|\partial_{1} v_{+}\right|^{2}+k_{+}^{2}\left|v_{+}\right|^{2}\right] d s \leq 2 k_{+} \Im \int_{\Gamma_{b}} \bar{v}_{+} \partial_{2} v_{+} d s,
\end{aligned}
$$

and

$$
\begin{aligned}
& \Im \int_{\Gamma_{a}} \bar{v}_{-} \partial_{2} v_{-} d s \leq 0, \quad \Re \int_{\Gamma_{a}} \bar{v}_{-} \partial_{2} v_{-} d s \geq 0, \\
& \int_{\Gamma_{a}}\left[\left|\partial_{2} v_{-}\right|^{2}-\left|\partial_{1} v_{-}\right|^{2}+k_{-}^{2}\left|v_{-}\right|^{2}\right] d s \leq-2 k_{-} \Im \int_{\Gamma_{a}} \bar{v}_{-} \partial_{2} v_{-} d s .
\end{aligned}
$$

The statements in this lemma concerning $v_{+}$were proved as in Lemma 6.1 in [7]. The statements regarding $v_{-}$follow from Remark 2.1.

Proof of Theorem 5.1. As in section 4, let $\eta<c<d<\rho$ and $a<0, b>B$. Also for convenience choose $a$ and $b$ so that $b-\beta=\beta-a \equiv \omega>0$.

Suppose that $u_{1}$ and $u_{2}$ are solutions of Problem (P). Then, by Remark 2.3, $u=u_{1}-u_{2} \in C^{1}\left(\mathbf{R}^{2}\right)$ and satisfies (2.3), the bound (2.8), the UPRC, and the DPRC. Also, by Theorem 3.1,

$$
\begin{array}{ll}
u(x)=\int_{E_{c}^{B}} u(y) k^{+}(y) G_{c}^{+}(x, y) d y+\int_{\Gamma_{c}} u(y) \frac{\partial G_{c}^{+}(x, y)}{\partial y_{2}} d s(y), \quad x \in U_{c}, \\
u(x)=\int_{E_{0}^{d}} u(y) k^{-}(y) G_{d}^{-}(x, y) d y-\int_{\Gamma_{d}} u(y) \frac{\partial G_{d}^{-}(x, y)}{\partial y_{2}} d s(y), \quad x \in \mathbf{R}^{2} \backslash \bar{U}_{d},
\end{array}
$$

and by Theorem 4.1, for some constants $C_{j} \geq 0, j=1,2,3$,

$$
\begin{aligned}
K_{A} \leq & C_{1}\left[\omega I_{A}^{+}(b)+\omega I_{A}^{-}(a)+L_{A}(b)-L_{A}(a)+R_{2}(A)\right] \\
& +C_{2}\left[J_{A}(a)-J_{A}(b)\right]+C_{3} R_{1}(A),
\end{aligned}
$$

where $J_{A}, I_{A}^{ \pm}, L_{A}$, and $K_{A}$ are given by (4.1) and (4.2). Clearly, for $j=1,2$,

$$
R_{j}(A)=O(1) \text { as } A \rightarrow \infty,
$$

and by (4.6),

$$
J_{A}(b)-J_{A}(a) \leq R_{1}(A)
$$

Now to make use of Lemma 5.1 and the bound (5.7), we define

$$
\begin{aligned}
& \text { (5.10) v(x)= } \int_{E_{c}^{B}(A)} u(y) k^{+}(y) G_{c}^{+}(x, y) d y+\int_{\Gamma_{c}(A)} u(y) \frac{\partial G_{c}^{+}(x, y)}{\partial y_{2}} d s(y), \quad x \in U_{c}, \\
& (5.11) v(x)=\int_{E_{0}^{d}(A)} u(y) k^{-}(y) G_{d}^{-}(x, y) d y-\int_{\Gamma_{d}(A)} u(y) \frac{\partial G_{d}^{-}(x, y)}{\partial y_{2}} d s(y), \quad x \in \mathbf{R}^{2} \backslash \bar{U}_{c} .
\end{aligned}
$$


Then, by (3.1)-(3.2), $\left.v\right|_{\Gamma_{B}} \in L_{2}\left(\Gamma_{B}\right) \cap B C\left(\Gamma_{B}\right)$ and $\left.v\right|_{\Gamma_{0}} \in L_{2}\left(\Gamma_{0}\right) \cap B C\left(\Gamma_{0}\right)$. Moreover, by Lemmas 3.1 and 3.2 and the equivalence of Lemmas 2.1(i)-(ii) and 2.2, $v$ satisfies (2.4), with $h=B$ and $\phi_{+}=\left.v\right|_{\Gamma_{B}}$, and satisfies (2.5) with $h=0$ and $\phi_{-}=\left.v\right|_{\Gamma_{0}}$. For $t \in \mathbf{R}$ set,

$$
\begin{array}{cl}
J_{A}^{\prime}(t)=\Im \int_{\Gamma_{t}(A)} \bar{v} \partial_{2} v d s, & J_{A}^{\prime \prime}(t)=\Im \int_{\Gamma_{t}} \bar{v} \partial_{2} v d s, \\
I_{A}^{ \pm \prime}(t)=\int_{\Gamma_{t}(A)}\left\{\left|\partial_{2} v\right|^{2}-\left|\partial_{1} v\right|^{2}+k_{ \pm}^{2}|v|^{2}\right\} d s, & I_{A}^{ \pm \prime \prime}=\int_{\Gamma_{t}}\left\{\left|\partial_{2} v\right|^{2}-\left|\partial_{1} v\right|^{2}+k_{ \pm}^{2}|v|^{2}\right\} d s, \\
L_{A}^{\prime}(t)=\Re \int_{\Gamma_{t}(A)} \bar{v} \partial_{2} v d s, & L_{A}^{\prime \prime}(t)=\Re \int_{\Gamma_{t}} \bar{v} \partial_{2} v d s .
\end{array}
$$

Then, by Lemma 5.2,

$$
\begin{array}{lll}
J_{A}^{\prime \prime}(b) \geq 0, & L_{A}^{\prime \prime}(b) \leq 0, & I_{A}^{+\prime \prime}(b) \leq 2 k_{+} J_{A}^{\prime \prime}(b) \\
J_{A}^{\prime \prime}(a) \leq 0, & L_{A}^{\prime \prime}(a) \geq 0, & I_{A}^{-\prime \prime}(a) \leq-2 k_{-} J_{A}^{\prime \prime}(a) .
\end{array}
$$

Hence, by the preceding and (5.7),

$$
\begin{aligned}
& K_{A} \leq C_{1}\{\omega {\left.\left[I_{A}^{+}(b)-I_{A}^{+\prime \prime}(b)\right]+\omega\left[I_{A}^{-}(a)-I_{A}^{-\prime \prime}(a)\right]+\left[L_{A}(b)-L_{A}^{\prime \prime}(b)\right]+\left[L_{A}^{\prime \prime}(a)-L_{A}(a)\right]\right\} } \\
&+ {\left[C_{2}+2 C_{1} \omega\left(k_{+}+k_{-}\right)\right]\left\{\left[J_{A}^{\prime \prime}(b)-J_{A}(b)\right]+\left[J_{A}(a)-J_{A}^{\prime \prime}(a)\right]\right\} } \\
&(5.12) \quad+\left[C_{3}+2 C_{1} \omega\left(k_{+}+k_{-}\right)\right] R_{1}(A)+C_{1} R_{2}(A) .
\end{aligned}
$$

Now note that

$$
K_{A}=\int_{-A}^{A}\left|w\left(x_{1}\right)\right|^{2} d x_{1},
$$

where

$$
\begin{aligned}
w\left(x_{1}\right)= & \left\{\int_{\eta}^{B}|u(x)|^{2}\left|k^{2}(x)-k_{+}^{2}\right| d x_{2}+\int_{0}^{\rho}|u(x)|^{2}\left|k^{2}(x)-k_{-}^{2}\right| d x_{2}\right. \\
& \left.+\left|u\left(x_{1}, c\right)\right|^{2}+\left|u\left(x_{1}, d\right)\right|^{2}\right\}^{1 / 2}, \quad x_{1} \in \mathbf{R},
\end{aligned}
$$

and note that by (3.3) and the Cauchy-Schwarz inequality, for $x \in \Gamma_{a}, \Gamma_{b}$,

$$
\begin{aligned}
|v(x)|, \quad|\nabla v(x)| & \leq C W_{A}\left(x_{1}\right), \\
|u(x)-v(x)|, \quad|\nabla u(x)-\nabla v(x)| & \leq C\left(W_{\infty}\left(x_{1}\right)-W_{A}\left(x_{1}\right)\right),
\end{aligned}
$$

where $C$ is a constant independent of $A$ and, for $0 \leq A \leq+\infty$,

$$
W_{A}\left(x_{1}\right)=\int_{-A}^{A}\left(1+\left|x_{1}-y_{1}\right|\right)^{-3 / 2} w\left(y_{1}\right) d y_{1}, \quad x_{1} \in \mathbf{R} .
$$

It follows that

$$
\begin{array}{ccc}
\mid I_{A}^{ \pm \prime}(t)- & I_{A}^{ \pm \prime \prime}(t)|, \quad| J_{A}^{\prime}(t)-J_{A}^{\prime \prime}(t) \mid, \quad & \left|L_{A}^{\prime}(t)-L_{A}^{\prime \prime}(t)\right| \\
\leq C \int_{\mathbf{R} \backslash[-A, A]}\left(W_{A}\left(x_{1}\right)\right)^{2} d x_{1}, & (t=a, b),
\end{array}
$$


where $C$ is a constant independent of $A$, and that

$$
\begin{aligned}
\mid I_{A}^{ \pm}(t)- & I_{A}^{ \pm \prime}(t)|, \quad| J_{A}(t)-J_{A}^{\prime}(t)|, \quad| L_{A}(t)-L_{A}^{\prime}(t) \mid \\
& \leq C \int_{-A}^{A}\left(W_{\infty}\left(x_{1}\right)-W_{A}\left(x_{1}\right)\right) W_{\infty}\left(x_{1}\right) d x_{1}, \quad(t=a, b),
\end{aligned}
$$

so that, from (5.12) for some constant $C_{0}>0$ and all $A>0$,

$$
\begin{aligned}
K_{A} \leq C_{0} & \left\{\int_{\mathbf{R} \backslash[-A, A]} W_{A}^{2}\left(x_{1}\right) d x_{1}+\int_{-A}^{A}\left(W_{\infty}\left(x_{1}\right)-W_{A}\left(x_{1}\right)\right) W_{\infty}\left(x_{1}\right) d x_{1}\right. \\
& \left.+\left|R_{1}(A)\right|+\left|R_{2}(A)\right|\right\} .
\end{aligned}
$$

Applying Lemma 5.1 to (5.13) we obtain that $w \in L_{2}(\mathbf{R})$, i.e., $u \in L_{2}\left(E_{B}\right) \cap L_{2}\left(\Gamma_{c}\right) \cap$ $L_{2}\left(\Gamma_{d}\right)$ and, for all $A_{0}>0$,

$$
\begin{array}{r}
\int_{E_{B}}|u|^{2} d x+\int_{\Gamma_{c}}|u|^{2} d s+\int_{\Gamma_{d}}|u|^{2} d s=\int_{-\infty}^{+\infty}|w|^{2} \\
\leq C_{0} \sup _{A>A_{0}}\left(\left|R_{1}(A)\right|+\left|R_{2}(A)\right|\right) .
\end{array}
$$

Since $u \in L_{2}\left(E_{B}\right) \cap L_{2}\left(\Gamma_{c}\right) \cap L_{2}\left(\Gamma_{d}\right)$, it follows from (5.5), (5.6), the bounds (3.1) and (3.2), and applications of Young's theorem that $u \in L_{2}\left(E_{a}^{b}\right)$ for any $a, b \in \mathbf{R}$ with $a<b$. Also, since $\nabla u \in B C\left(E_{a}^{b}\right)$ so that $u$ is uniformly continuous in $\overline{E_{a}^{b}}$, it follows that $u(x) \rightarrow 0$ as $x_{1} \rightarrow \infty$ uniformly in $x_{2}$ for $a \leq x_{2} \leq b$ for any real numbers $a<b$. Also, noting Lemma 2.2, it follows that $R_{j}(A) \rightarrow 0$ as $A \rightarrow \infty, j=1,2$, and thus, from (5.14), that $u=0$ in $E_{B}$ and on $\Gamma_{c} \cup \Gamma_{d}$; and hence, from (5.5) and (5.6), that $u \equiv 0$ in $\mathbf{R}^{2}$.

6. Existence of solution. In this section existence of a solution for Problem (P) will be established by making use of general results on the solvability of the system of second-kind integral equations

$$
\psi_{i}=\phi_{i}+\sum_{j=1}^{N} K_{i j} \psi_{j}, \quad i=1, \ldots, N,
$$

in which $\phi_{i} \in Y_{i}:=B C\left(\bar{\Omega}_{i}\right)$ is assumed known, $\psi_{i} \in Y_{i}$ is to be determined, and $K_{i j}: Y_{j} \rightarrow Y_{i}$ is the integral operator defined by

$$
K_{i j} \psi(x)=\int_{\Omega_{j}} k_{i j}(x, y) \psi(y) d \mu_{j}(y), \quad x \in \bar{\Omega}_{i},
$$

$i, j=1, \ldots, N$. Here $\Omega_{j}$ is an open subset of $\mathbf{R}^{n_{j}}\left(n_{j} \geq 1\right)$ and $d \mu_{j}$ is $n_{j}$-dimensional Lebesgue measure. The function $k_{i j}: \bar{\Omega}_{i} \times \Omega_{j} \rightarrow \mathbf{C}$ is assumed to take the form, for some $M \in \mathbf{N}$,

$$
k_{i j}(x, y)=\sum_{m=1}^{M} k_{i j}^{(m)}(x, y) z_{j}^{(m)}(y)
$$


where $z_{j}^{(m)} \in X_{j}:=L_{\infty}\left(\Omega_{j}\right)$ and $k_{i j}^{(m)}(x, \cdot) \in L_{1}\left(\Omega_{j}\right)$ for every $x \in \bar{\Omega}_{i}(i, j=$ $1, \ldots, N, m=1, \ldots, M)$. We assume that the following conditions on $k_{i j}^{(m)}$ and $\Omega_{j}$ hold:

(C.1) $\sup _{x \in \bar{\Omega}_{i}} \int_{\Omega_{j}}\left|k_{i j}^{(m)}(x, y)\right| d \mu_{j}(y)<\infty$ and, for all $x \in \bar{\Omega}_{i}$,

$$
\int_{\Omega_{j}}\left|k_{i j}^{(m)}(x, y)-k_{i j}^{(m)}\left(x^{\prime}, y\right)\right| d \mu_{j}(y) \rightarrow 0
$$

as $x^{\prime} \rightarrow x$ with $x^{\prime} \in \bar{\Omega}_{i}(i, j=1, \ldots, N, m=1, \ldots, M)$.

(C.2) For some $n_{0} \leq \min _{j} n_{j}$ and $i=1, \ldots, N$, there exists $a_{j}^{(i)} \in \mathbf{R}^{n_{i}}, j=$ $1, \ldots, n_{0}$, and a bounded set $\omega_{i} \subset \Omega_{i}$ such that

(i) $\Omega_{i}=\bigcup_{P \in \mathbf{Z}^{n_{0}}} \omega_{i}^{(P)}$, where $\omega_{i}^{(P)}:=\omega_{i}+\sum_{j=1}^{n_{0}} a_{j}^{(i)} p_{j}$, for $P=\left(p_{1}, \ldots, p_{n_{0}}\right) \in$ $\mathbf{Z}^{n_{0}}$

(ii) $\omega_{i}^{(Q)} \cap \omega_{i}^{(P)}=\emptyset$ for $Q, P \in \mathbf{Z}^{n_{0}}, Q \neq P$;

(iii) $k_{i j}^{(m)}\left(x+a_{l}^{(i)}, y+a_{l}^{(j)}\right)=k_{i j}^{(m)}(x, y), x \in \bar{\Omega}_{i}, y \in \Omega_{j}, i, j=1, \ldots, N, l=$ $1, \ldots, n_{0}$, $m=1, \ldots, M$.

Let $X$ and $Y$ denote the product spaces $X:=\prod_{j=1}^{N} X_{j}$ and $Y:=\prod_{j=1}^{N} Y_{j} \subset X$. Let $\phi=\left(\phi_{1}, \ldots, \phi_{N}\right)^{t}, \psi=\left(\psi_{1}, \ldots, \psi_{N}\right)^{t} \in Y$, where $(\cdot, \ldots, \cdot)^{t}$ denotes the transpose of $(\cdot, \ldots, \cdot)$. For $m=1, \ldots, M$, define the matrix operator $K^{(m)}$ on $X$ by

$$
K^{(m)}=\left(\begin{array}{ccc}
K_{11}^{(m)} & \cdots & K_{1 N}^{(m)} \\
& \ddots & \\
K_{N 1}^{(m)} & \cdots & K_{N N}^{(m)}
\end{array}\right),
$$

where $K_{i j}^{(m)}: X_{j} \rightarrow X_{i}$ is the integral operator defined by (6.2) with $K_{i j}, k_{i j}$ replaced by $K_{i j}^{(m)}, k_{i j}^{(m)}$. For $z=\left(z_{1}, \ldots, z_{N}\right)^{t} \in X$ define $\hat{z}$ by

$$
\hat{z}=\left(\begin{array}{ccc}
z_{1} & \cdots & 0 \\
& \ddots & \\
0 & \cdots & z_{N}
\end{array}\right)
$$

and for $m=1, \ldots, M$ and $z \in X$, define $K_{z}^{(m)}: Y \rightarrow Y$ by

$$
K_{z}^{(m)} \psi=K^{(m)}(\hat{z} \psi), \quad \psi \in Y .
$$

For $w=\left(w^{(1)}, \ldots, w^{(M)}\right) \in X^{M}$ let $K_{w}$ denote the matrix integral operator

$$
K_{w}=\sum_{m=1}^{M} K_{w^{(m)}}^{(m)}
$$

Then (6.1) can be abbreviated as

$$
\psi=\phi+K_{z} \psi,
$$

where $z=\left(z^{(1)}, \ldots, z^{(M)}\right)$ and $z^{(m)}=\left(z_{1}^{(m)}, \ldots, z_{N}^{(m)}\right)^{t}, m=1, \ldots, M$. 
For $j=1, \ldots, N, i=1, \ldots, n_{0}$, define the translation operator $T_{a_{j}^{(i)}}: X_{j} \rightarrow X_{j}$ by

$$
T_{a_{j}^{(i)}} \psi(x)=\psi\left(x-a_{j}^{(i)}\right), \quad x \in \Omega_{j},
$$

and for $a=\left(a_{1}, \ldots, a_{N}\right) \in \tau:=\left\{\left(a_{l}^{(1)}, \ldots, a_{l}^{(N)}\right)^{t} \mid l=1, \ldots, n_{0}\right\}$, define the matrix operator $T_{a}: X \rightarrow X$ by

$$
T_{a}=\left(\begin{array}{ccc}
T_{a_{1}} & & 0 \\
& \ddots & \\
0 & & T_{a_{N}}
\end{array}\right) .
$$

Then, by (C.2) (iii), $T_{a} K^{(m)}=K^{(m)} T_{a}, a \in \tau, m=1, \ldots, M$. Let $B(Y)$ denote the Banach space of bounded linear operators on $Y$ and $I$ the identity matrix operator on $Y$. The following results have been proved in [10], extending the results of [9] for single integral equations to systems of integral equations.

Theorem 6.1. Suppose that (C.1) and (C.2) are satisfied, that $W \subset X^{M}$ is weak* sequentially compact, that $T_{a} W:=\left\{\left(T_{a} z^{(1)}, \ldots, T_{a} z^{(M)}\right) \mid\left(z^{(1)}, \ldots, z^{(M)}\right) \in W\right\}=W$, $a \in \tau$, and that $I-K_{z}$ is injective for all $z \in W$. Then $\left(I-K_{z}\right)^{-1}$ exists as an operator on the range space $\left(I-K_{z}\right) Y$ for all $z \in W$ and

$$
\sup _{z \in W}\left\|\left(I-K_{z}\right)^{-1}\right\|<\infty .
$$

Also, if for every $z \in W$ there exists a sequence $\left(z_{j}\right) \subset W$ such that $\left(z_{j}\right)$ converges weak* to $z$ in $X$ and

$$
\text { for all } j, \quad I-K_{z_{j}} \quad \text { injective } \Longrightarrow I-K_{z_{j}} \text { surjective, }
$$

then $I-K_{z}$ is surjective also for each $z \in W$ so that $\left(I-K_{z}\right)^{-1} \in B(Y)$.

TheOREM 6.2. If (C.1) and (C.2) are satisfied, $z=\left(\left(z_{1}^{(1)}, \ldots, z_{N}^{(1)}\right)^{t}, \ldots\right.$, $\left.\left(z_{1}^{(M)}, \ldots, z_{N}^{(M)}\right)^{t}\right) \in X^{M}$, and for some constants $\lambda_{j}^{(m)} \in \mathbf{C}, j=1, \ldots, N, m=$ $1, \ldots, M$, it holds that

$$
\operatorname{ess} \sup _{|x| \geq A, x \in \Omega_{j}}\left|z_{j}^{(m)}(x)-\lambda_{j}^{(m)}\right| \rightarrow 0
$$

as $A \rightarrow \infty$, then

$$
I-K_{\lambda}, \quad I-K_{z} \text { injective } \Rightarrow I-K_{z} \text { surjective, } \quad\left(I-K_{z}\right)^{-1} \in B(Y),
$$
where $\lambda=\left(\left(\lambda_{1}^{(1)}, \ldots, \lambda_{N}^{(1)}\right)^{t}, \ldots,\left(\lambda_{1}^{(M)}, \ldots, \lambda_{N}^{(M)}\right)^{t}\right)$.

To apply Theorems 6.1 and 6.2 to show the existence of a solution to Problem $(\mathrm{P})$, we choose $\lambda, c$, and $d$ so that $0 \leq c<d \leq B, k_{-}(d-\lambda)=k_{+}(\lambda-c)$, and $2(d-c) k_{+} k_{-}<\pi\left(k_{+}+k_{-}\right)$. It then follows from Theorem 3.2 that Problem $(\mathrm{P})$ and the integral equation problems (3.10) and (3.11) are equivalent.

Let $N=4, n_{0}=1, \Omega_{1}=E_{\lambda}^{B} \subset \mathbf{R}^{2}, \Omega_{2}=E_{0}^{\lambda} \subset \mathbf{R}^{2}, \Omega_{3}=\Omega_{4}=\mathbf{R}, \omega_{1}=$ $\left\{x \in \Omega_{1} \mid 0 \leq x_{1}<B, \lambda<x_{2}<B\right\}, \omega_{2}=\left\{x \in \Omega_{2} \mid 0 \leq x_{1}<B, 0<x_{2}<\lambda\right\}$, $\omega_{3}=\omega_{4}=[0, B), a_{1}^{(1)}=a_{1}^{(2)}=(B, 0)$, and $a_{1}^{(3)}=a_{1}^{(4)}=B$. Define $\tilde{y}:=y$ and $\hat{y}:=y$ for $y \in \mathbf{R}^{2}$ and $\tilde{y}:=(y, c)$ and $\hat{y}:=(y, d)$ for $y \in \mathbf{R}$. Let $\chi(t)=1, t>0$, $=0, t<0$, and let $M=2, k_{i j}^{(1)}(x, y)=G_{c}^{+}(\hat{x}, y) \chi\left(y_{2}-c\right)$ for all $x \in \Omega_{i}, y \in \Omega_{j}$, 
$\hat{x} \neq y, i=1,3, j=1,2, k_{i j}^{(1)}(x, y)=G_{d}^{-}(\tilde{x}, y) \chi\left(d-y_{2}\right)$ for all $x \in \Omega_{i}, y \in \Omega_{j}, \tilde{x} \neq y$, $i=2,4, j=1,2, k_{i 4}^{(1)}(x, y)=\partial G_{c}^{+}(\hat{x}, z) /\left.\partial z_{2}\right|_{z=\tilde{y}}$ for all $x \in \Omega_{i}, y \in \Omega_{4}, i=1,3$, $k_{i 3}^{(1)}(x, y)=\partial G_{d}^{-}(\tilde{x}, z) /\left.\partial z_{2}\right|_{z=\hat{y}}$ for all $x \in \Omega_{i}, y \in \Omega_{3}, i=2,4, k_{i j}^{(1)}(x, y)=0$ for all $x \in \Omega_{i}, y \in \Omega_{j}$, with $i=1,3, j=3$, or $i=2,4, j=4$. Let $k_{i j}^{(2)}=k_{i j}^{(1)}, i=2,4$, $j=1,2,=0$, otherwise. Then conditions (C.1) and (C.2) are satisfied with these choices of $k_{i j}^{(m)}$ and $\Omega_{j}(i, j=1,2,3,4, m=1,2)$. Set $w_{j}^{(1)}(y)=k^{+}(y)$ for $y \in \Omega_{j}$, $j=1,2, w_{3}^{(1)}(y)=-1, y \in \Omega_{3}, w_{4}^{(1)}(y)=1, y \in \Omega_{4}$, and set $w^{(1)}=\left(w_{1}^{(1)}, \ldots, w_{4}^{(1)}\right)^{t}$, $w^{(2)}=\left(k_{+}^{2}-k_{-}^{2}\right)(1,1,0,0)^{t}$. Then the integral equations (3.10) and (3.11) can be written as the $4 \times 4$ matrix system

$$
\left(I-K_{w}\right) \psi=\phi, \quad \psi=\left(\psi_{1}, \ldots, \psi_{4}\right)^{t}, \quad \phi=\left(\phi_{1}, \ldots, \phi_{4}\right)^{t} \in Y,
$$

where $w=\left(w^{(1)}, w^{(2)}\right), K_{w}$ is defined by (6.5), (6.4), and (6.3), $\phi_{2}=\phi_{4}=0, \phi_{j}(y)=$ $u^{i}(\hat{y})+u^{r}(\hat{y}), y \in \bar{\Omega}_{j}, j=1,3$, and $\psi_{3}, \psi_{4} \in B C(\mathbf{R})$ are defined by $\psi_{3}(y)=\psi_{2}(\hat{y})$, $\psi_{4}(y)=\psi_{1}(\tilde{y}), y \in \mathbf{R}$.

THEOREM 6.3. Assume that (A3) holds or that (A4) and (A5) hold and that $k_{-}(d-\lambda)=k_{+}(\lambda-c)$ and $2(d-c) k_{+} k_{-}<\pi\left(k_{+}+k_{-}\right)$. Then $\left(I-K_{w}\right)^{-1} \in B(Y)$ so that the system of integral equations (6.7) has a unique solution $\psi \in Y$. Furthermore, for any $L>0$, there is a constant $C>0$ depending only on $L, k_{ \pm}, c, d, \eta, \rho, B, \lambda_{1}$, and $\lambda_{2}$, in the case that (A3) is satisfied, or on $L, k_{ \pm}, c, d, \eta, \rho, B, \beta$, and $\lambda_{3}$, in the case that (A4) and (A5) are satisfied such that, provided $\|k\|_{\infty} \leq L,\left\|\left(I-K_{w}\right)^{-1}\right\| \leq C$ so that $\|\psi\| \leq C\|\phi\|$.

Proof. Theorem 6.3 is proved by means of Theorems 6.1 and 6.2. To this end, suppose without loss of generality that $L>k_{ \pm}^{2}+\lambda_{1}$ and set

$$
\begin{aligned}
Q=Q_{3}:= & \left\{\mu \in L_{\infty}\left(E_{B}\right)\left|\Im \mu \geq 0, \Im \mu(x) \geq \lambda_{1}, x \in E_{\eta}^{\rho}, \Im \mu(x) \geq \lambda_{2}\right| \mu(x)-k_{+}^{2} \mid,\right. \\
& \left.x \in E_{\eta}^{B}, \Im \mu(x) \geq \lambda_{2}\left|\mu(x)-k_{-}^{2}\right|, x \in E_{0}^{\rho},\|\mu\|_{\infty} \leq L^{2}\right\}
\end{aligned}
$$

in the case that (A3) is satisfied. In the case that (A4) and (A5) are satisfied suppose without loss of generality that $L^{2}>k_{ \pm}^{2}+\lambda_{3}$ and set

$$
\begin{aligned}
& Q=Q_{4}:=\left\{\mu \in L_{\infty}\left(\mathbf{R}^{2}\right) \mid \Im \mu \geq 0, \mu(x)=k_{+}^{2}, x \in U_{B}, \mu(x)=k_{-}^{2}, x \in \mathbf{R}^{2} \backslash \bar{U},\right. \\
& \|\mu\|_{\infty} \leq L^{2}, \operatorname{ess}_{\inf _{x \in U_{\beta}}} \Re\left[\mu\left(x+e_{2} h\right)-\mu(x)\right] \geq 0,
\end{aligned}
$$

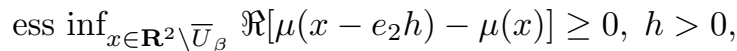

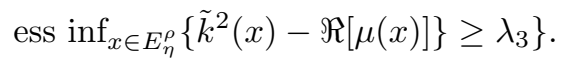

Define $W^{(1)} \subset X$ by

$$
W^{(1)}=\left\{\left(\left.\mu\right|_{\Omega_{1}}-k_{+}^{2},\left.\mu\right|_{\Omega_{2}}-k_{+}^{2},-1,1\right)^{t} \mid \mu \in Q\right\}
$$

and $W \subset X^{2}$ by

$$
W=\left\{\left(w^{(1)},\left(k_{+}^{2}-k_{-}^{2}, k_{+}^{2}-k_{-}^{2}, 0,0\right)^{t}\right) \mid w^{(1)} \in W^{(1)}\right\} .
$$

Then $T_{a} W=W$ for $a \in \tau=\left\{\left(a_{1}^{(1)}, \ldots, a_{1}^{(4)}\right)\right\}$. Also, it follows easily from Theorems 3.2 and 5.1 that $I-K_{z}$ is injective for all $z \in W$.

Next, we show that $W$ is weak* sequentially compact. In view of the definition of $W$ it is sufficient to show that $W^{(1)} \subset X$ is weak* sequentially compact. Further, 
in view of the definition of $W^{(1)}$, it is sufficient to show that $Q$ is weak* sequentially compact, where $Q=Q_{3} \subset L_{\infty}\left(E_{B}\right)$ in the case that (A3) is satisfied and $Q=Q_{4} \subset$ $L_{\infty}\left(\mathbf{R}^{2}\right)$ in the case that (A4) and (A5) are satisfied.

Since $Q$ is bounded it follows from the Alaoglu theorem [16, p. 60] that $Q$ is weak* sequentially compact if it is weak* sequentially closed. In the case $Q=Q_{3}$, the fact that $Q$ is weak* sequentially closed follows from Lemma 2.13 in [9], since the sets $\left\{w \in \mathbf{C}|| w \mid \leq L^{2}, \Im w \geq \lambda_{1}\right\}$ and $\left\{w \in \mathbf{C}|| w\left|\leq L^{2}, \Im w \geq 0, \Im w \geq \lambda_{2}\right| w-k_{*}^{2} \mid\right\}$, for $k_{*}=k_{+}$and $k_{-}$, are compact and convex.

In the case $Q=Q_{4}$, in order to see that $Q$ is weak* sequentially compact consider a sequence $\left(\mu_{j}\right) \subset Q$. Since $\left(\mu_{j}\right)$ is bounded, it follows from the Alaoglu theorem [16, p. 60] that there is an element $\mu \in L_{\infty}\left(\mathbf{R}^{2}\right)$ and a subsequence of $\left(\mu_{j}\right)$, denoted simply by itself, such that $\left(\mu_{j}\right)$ converges weak* to $\mu$ in $L_{\infty}\left(\mathbf{R}^{2}\right)$ and $\|\mu\|_{\infty} \leq L^{2}$. Thus, for all $\xi \in L_{1}\left(\mathbf{R}^{2}\right)$,

$$
\int_{\mathbf{R}^{2}} \mu_{j} \xi d x \rightarrow \int_{\mathbf{R}^{2}} \mu \xi d x,
$$

as $j \rightarrow \infty$ and, in particular, (6.8) holds if $\xi$ is the characteristic function of any bounded measurable subset of $\mathbf{R}^{2}$. This and the fact that $\mu_{j} \in Q, j=1,2, \ldots$, implies that $\Im \mu \geq 0$ in $\mathbf{R}^{2}, \mu(x)=k_{+}^{2}$ for $x \in U_{B}, \mu(x)=k_{-}^{2}$ for $x \in \mathbf{R}^{2} \backslash \bar{U}$, $\operatorname{ess} \inf _{x \in E_{\eta}^{\rho}}\left\{\tilde{k}^{2}(x)-\Re[\mu(x)]\right\} \geq \lambda_{2}$, and

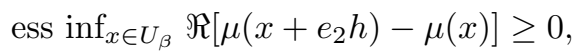

$$
\begin{aligned}
& \text { ess } \inf _{x \in \mathbf{R}^{2} \backslash \bar{U}_{\beta}} \Re\left[\mu\left(x-e_{2} h\right)-\mu(x)\right] \geq 0
\end{aligned}
$$

for all $h>0$. Hence $\mu \in Q$ and $\left(\mu_{j}\right)$ converges weak* to $\mu$ in $Q$. Thus $Q$ is weak* sequentially compact.

Finally, let $z=\left(z^{(1)}, z^{(2)}\right) \in W$. Then, for some $\mu \in Q, z^{(1)}=\left(\left.\mu\right|_{\Omega_{1}}-k_{+}^{2},\left.\mu\right|_{\Omega_{2}}-\right.$ $\left.k_{+}^{2},-1,1\right)^{t}$ and $z^{(2)}=\left(k_{+}^{2}-k_{-}^{2}\right)(1,1,0,0)^{t}$. For $j=1,2, \ldots$, set

$$
\mu_{j}(x)= \begin{cases}\mu^{*}(x) & \text { for }\left|x_{1}\right|>j \\ \mu(x) & \text { for }\left|x_{1}\right| \leq j\end{cases}
$$

where $\mu^{*} \equiv i \lambda_{1}$ in the case that (A3) is satisfied, $\mu^{*}=k_{+}^{2}$ in $U_{B},=k_{-}^{2}$ in $\mathbf{R}^{2} \backslash \bar{U},=$ $\min \left(k_{-}^{2}, k_{+}^{2}\right)-\lambda_{2}$ in $E_{B}$, in the case that (A4) and (A5) are satisfied. Then $\mu^{*}, \mu_{j} \in Q$, and setting $z_{j}^{(1)}=\left(\left.\mu_{j}\right|_{\Omega_{1}}-k_{+}^{2},\left.\mu_{j}\right|_{\Omega_{2}}-k_{+}^{2},-1,1\right)^{t}$ and $z_{j}=\left(z_{j}^{(1)}, z^{(2)}\right), j=1,2, \ldots$, it is easy to see that $\left(z_{j}\right)$ converges weak $*$ to $z$. Define

$$
\begin{aligned}
z^{*} & =\left(\left(\left.\mu^{*}\right|_{\Omega_{1}}-k_{+}^{2},\left.\mu^{*}\right|_{\Omega_{2}}-k_{+}^{2},-1,1\right)^{t}, z^{(2)}\right) \\
& =\left(\left(\lambda^{*}, \lambda^{*},-1,1\right)^{t},\left(k_{+}^{2}-k_{-}^{2}, k_{+}^{2}-k_{-}^{2}, 0,0\right)^{t}\right),
\end{aligned}
$$

where $\lambda^{*} \in \mathbf{C}$ is given by $\lambda^{*}=i \lambda_{1}-k_{+}^{2}$ in the case that (A3) is satisfied and by $\lambda^{*}=\min \left(k_{-}^{2}, k_{+}^{2}\right)-\lambda_{2}-k_{+}^{2}$ in the case that (A4) and (A5) are satisfied. Since $z^{*} \in W$ so that $I-K_{z^{*}}$ is injective, it follows from Theorem 6.2 that $I-K_{z_{j}}$ injective implies $I-K_{z_{j}}$ surjective, for $j=1,2, \ldots$.

All the assumptions in Theorem 6.1 have been verified so Theorem 6.3 follows from Theorem 6.1.

TheOREm 6.4. Assume that (A3) holds or that (A4) and (A5) hold. Then Problem (P) has exactly one solution. Further, for any $L>0$, there exists a constant $C>0$ depending only on $L, k_{ \pm}, \eta, \rho, B, \lambda_{1}$, and $\lambda_{2}$ in the case that (A3) is satisfied, or 
on $L, k_{ \pm}, \eta, \rho, B, \beta$, and $\lambda_{3}$ in the case that (A4) and (A5) are satisfied such that, provided $\|k\|_{\infty} \leq L$,

$$
|u(x)| \leq C\left(1+\left|x_{2}\right|\right)^{1 / 2}, \quad x \in \mathbf{R}^{2} .
$$

Proof. The existence of a unique solution to Problem (P) follows from Theorems $3.1,3.2$, and 6.3. To derive the estimate (6.9) we note from the equivalence of (i) and (ii) in Lemma 2.1 that, for $h>B$,

$$
u^{\mathrm{s}}(x)=2 \int_{\Gamma_{h}} \frac{\partial \Phi(x, y)}{\partial y_{2}} u^{\mathrm{s}}(y) d s(y), \quad x \in U_{h} .
$$

It follows from (2.6) and (6.10) (see [5]) that

$$
\left|u^{\mathrm{s}}(x)\right| \leq C\left(1+\left(x_{2}-B\right)\right)^{1 / 2} \sup _{x \in \Gamma_{B}}|u(x)|, \quad x \in U_{B},
$$

for some constant $C>0$ dependent only on $k_{+}$, which together with Theorem 6.3 implies the estimate (6.9) for $x \in U$. The estimate (6.9) for $x \in \mathbf{R}^{2} \backslash U$ can be proved similarly by using Lemma 2.2 .

Appendix: Guided waves. By a guided wave we mean a solution of the homogeneous problem which has its energy localized in or near the layer $E_{B}$. Precisely, for $a<b$, let $D(a, b)=\left\{x \in \mathbf{R}^{2} \mid a<x_{1}<b\right\}$. Then our definition is as follows.

Definition A.1. Call $v \in C^{1}\left(\mathbf{R}^{2}\right)$ a guided wave if $v \not \equiv 0, v$ satisfies (2.3), $v$ is bounded in $E_{-h}^{h}$ for every $h>0$,

$$
\sup _{n \in \mathbf{Z}} \int_{D(n, n+1)}\left(|v|^{2}+|\nabla v|^{2}\right) d x<\infty,
$$

and

$$
c_{h}:=\sup _{n \in \mathbf{Z}} \int_{D(n, n+1) \backslash E_{-h}^{h}}\left(|v|^{2}+|\nabla v|^{2}\right) d x \rightarrow 0
$$

as $h \rightarrow \infty$.

Remark A.1. In the case when the scatterer is a diffraction grating, i.e., $k$ is periodic in the $x_{1}$-direction with some period $L$, it is usual to assume that $v$ is correspondingly quasi periodic (i.e., that $v(x) \exp \left(-i k_{+} \cos \theta x_{1}\right)$ is periodic). Then (A.1) and (A.2) reduce to the condition that

$$
\int_{D(0, L)}\left(|v|^{2}+|\nabla v|^{2}\right) d x<\infty
$$

i.e., that the energy is finite in a single period of the grating (cf. Bonnet-Bendhia and Starling [3]).

Remark A.2. Conditions (A.1) and (A.2) are satisfied if $v$ decreases rapidly enough in the vertical direction, in particular, if for some constants $C>0$ and $p>1 / 2$,

$$
|v(x)| \leq C\left(1+\left|x_{2}\right|\right)^{-p}, \quad x \in \mathbf{R}^{2} .
$$

The following result follows from Theorem 8.1 in [7] and Remark 2.1.

THEOREM A.1. If $v$ is a guided wave, then $v$ satisfies the UPRC for wavenumber $k_{+}$and the DPRC for wavenumber $k_{-}$. 


\section{REFERENCES}

[1] G. BAO, Finite element approximation of time harmonic waves in periodic structures, SIAM J. Numer. Anal. 32 (1995), pp. 1155-1169.

[2] G. BaO, D. C. Dobson, And J. A. Cox, Mathematical studies in rigorous grating theory, J. Opt. Soc. Amer. A 12 (1995), pp. 1029-1042.

[3] A.-S. Bonnet-Bendhia And F. Starling, Guided waves by electromagnetic gratings and nonuniqueness examples for the diffraction problem, Math. Methods Appl. Sci., 17 (1994), pp. 305-338.

[4] S. N. Chandler-Wilde, Boundary value problems for the Helmholtz equation in a half-plane, in Proceedings, Third Int. Conf. on Mathematical and Numerical Aspects of Wave Propagation, G. Cohen, L. Halpern, and P. Joly, eds., Proceedings Appl. Math 50, SIAM, Philadelphia, PA, 1995, pp. 188-197.

[5] S. N. Chandler-Wilde, The impedance boundary value problem for the Helmholtz equation in a half-plane, Math. Methods Appl. Sci., 20 (1997), pp. 813-840.

[6] S. N. Chandler-Wilde And C. R. Ross, Scattering by rough surfaces: The Dirichlet problem for the Helmholtz equation in a non-locally perturbed half-plane, Math. Methods Appl. Sci., 19 (1996), pp. 959-976.

[7] S. N. Chandler-Wilde AND B. Zhang, Electromagnetic scattering by an inhomogeneous conducting or dielectric layer on a perfectly conducting plate, Proc. Roy. Soc. London Ser. A, 454 (1998), pp. 519-542.

[8] S. N. Chandler-Wilde And B. Zhang, A uniqueness result for scattering by infinite rough surfaces, SIAM J. Appl. Math., 58 (1998), pp. 1774-1790.

[9] S. N. Chandler-Wilde And B. Zhang, On the solvability of a class of second kind integral equations on unbounded domains, J. Math. Anal. Appl., 214 (1997), pp. 482-502.

[10] S. N. Chandler-Wilde and B. Zhang, A Generalized Collectively Compact Operator Theory with an Application to Integral Equations on Unbounded Domains, in preparation.

[11] X. Chen And A. Friedman, Maxwell's equations in a periodic structure, Trans. Amer. Math. Soc., 323 (1991), pp. 465-507.

[12] D. Colton And R. KRess, Integral Equation Methods in Scattering Theory, John Wiley, New York, 1983.

[13] D. Colton And R. Kress, Inverse Acoustic and Electromagnetic Scattering Theory, 2nd ed., Springer-Verlag, Berlin, 1998.

[14] C. Macaskill and P. CaO, A new treatment of rough surface scattering, Proc. Roy. Soc. London Ser A, 452 (1996), pp. 2593-2612.

[15] J. A. DeSanto and P. A. Martin, On the derivation of boundary integral equations for scattering by an infinite one-dimensional rough surface, J. Acoust. Soc. Am. 102 (1997), pp. $67-77$.

[16] C. L. DeVito, Functional Analysis, Academic Press, New York, 1978.

[17] D. Dobson and A. Friedman, The time-harmonic Maxwell equations in a doubly periodic structure, J. Math. Anal. Appl. 166 (1992), pp. 507-528.

[18] D. Gilbarg and N.S. Trudinger, Elliptic Partial Differential Equations of Second Order, 2nd ed., Springer-Verlag, Berlin, 1983.

[19] A. KIrsch, Diffraction by periodic structures, in Inverse Problems in Mathematical Physics, L. Paivarinta and E. Somersalo, eds., Lecture Notes in Phys. 422, Springer-Verlag, 1993, pp. $87-102$.

[20] A. KIRSCH, An inverse problem for periodic structures, in Inverse Scattering and Potential Problems in Mathematical Physics, R.E. Kleinman, R. Kress, and E. Martensen, eds., Peter Lang, Frankfurt, 1995, pp. 75-93.

[21] D. A. KAPP AND G. S. BRown, A new numerical method for rough surface scattering calculations, IEEE Trans. Antennas and Propagation, 44 (1996), pp. 711-721.

[22] J. C. Nedelec AND F. STARLing, Integral equation methods in a quasi-periodic diffraction problem for the time-harmonic Maxwell's equations, SIAM J. Math. Anal. 22 (1991), pp. 16791701.

[23] R. Petit, Electromagnetic Theory of Gratings, Springer-Verlag, Berlin, 1980.

[24] H. W. Schürmann, V. S. Serov, and Yu. V. Shestopalov, On the theory of TE-polarized waves in a linear three-layer structure, Electromagnetic Waves and Electronic Systems, 1 (1997), pp. 49-59.

[25] B. Strycharz, An acoustic scattering problem for a periodic, inhomogeneous media, Math. Methods Appl. Sci., 21 (1998), pp. 969-983.

[26] L. Tsang, C. H. Chan, K. Pak, and H. Sangani, Monte-Carlo simulations of large-scale problems of random rough surface scattering and applications to grazing incidence with 
the BMIA/canonical grid method, IEEE Trans. Antennas and Propagation, 43 (1995), pp. $851-859$.

[27] G. VAinikKo, Multidimensional Weakly Singular Integral Equations, Springer-Verlag, Berlin, 1993.

[28] G. VAinikko, Fast Solvers of the Lippmann-Schwinger Equation, Research reports A387, Institute of Mathematics, Helsinki University of Technology, Finland 1997.

[29] R. L. Wagner, J. M. Song, And W. C. Chew, Monte-Carlo simulation of electromagnetic scattering from two-dimensional random rough surfaces, IEEE Trans. Antennas and Propagation, 45 (1997), pp. 235-246.

[30] Y. XU, Radiation condition and scattering problem for time-harmonic acoustic waves in a stratified medium with a nonstratified inhomogeneity, IMA J. Appl. Math., 54 (1995), pp. 9-29.

[31] Bo Zhang and S. N. Chandler-Wilde, Acoustic scattering by an inhomogeneous layer on a rigid plate, SIAM J. Appl. Math. 58 (1998), pp. 1931-1950. 